\title{
An approach to plane algebroid branches
}

\author{
Evelia R. García Barroso and Arkadiusz Płoski
}

August 1, 2018

\begin{abstract}
Our aim is to reprove the basic results of the theory of branches of plane algebraic curves over algebraically closed fields of arbitrary characteristic. We do not use the Hamburger-Noether expansions. Our basic tool is the logarithmic distance on the set of branches satisfying the strong triangle inequality which permits to make calculations directly on the equations of branches.

What can be explained on fewer principles is explained needlessly by more. William of Ockham (1280-1349) 1
\end{abstract}

\section{Introduction}

We present a new approach to the theory of plane algebroid branches over an algebraically closed field of arbitrary characteristic. We prove the structure theorem for the semigroup of plane branches, the fundamental theorems of the Abhyankar-Moh theory, the intersection formula and the existence of a branch with given semigroup. These results are well-known (at least in characteristic 0) but our proofs are new. In constrast to classical treatments of the subject given by Ancochea (1947), Lejeune-Jalabert (1973), Moh (1973), Angermüller (1977), Russel (1980) and Campillo (1980) we do not use the

2000 Mathematics Subject Classification: Primary 32S55; Secondary $14 \mathrm{H} 20$.

Key words and phrases: plane algebroid curve, branch, semigroup associated with a branch, key polynomials, logarithmic distance, Abhyankar-Moh theory.

The first-named author was partially supported by the Spanish Project PNMTM 2007-64007.

${ }^{1}$ Quoted after Samuel E. Stumpf. Socrates to Sartre. A History of Philosophy. Mc Graw-Hill, Inc. 1993. 
quadratic transformations. To avoid the Hamburger-Noether expansions we base our approach on the direct construction of key polynomials (the notion introduced by MacLane (1936)) given by Seidenberg in his $\mathrm{PhD}$ thesis on the valuation ideals in polynomial rings. As far as we know the Seidenberg article of 1945 is the first publication in which appears the God-given inequality $n_{k} \overline{\beta_{k}}<\overline{\beta_{k+1}}$ (we use the notation introduced by Zariski).

In all this paper we use the strong triangle inequality (STI) proved by the second author in 1985. It allows to give simple proofs of all basic properties of key polynomials in any characteristic. Using the STI we prove the Abhyankar-Moh irreducibility criterion in arbitrary characteristic, the description of branches with given semigroup and the Merle-Granja factorization theorem.

A plane algebroid branch may be given either by an irreducible equation $f(x, y)=0$ or by a parametrization $x=\phi(t), y=\psi(t)$. The treatments of the subject which use the Hambuger-Noether expansions (or Puiseux' expansion in the case of characteristic 0) are based on the interplay between the equations and the parametrizations of branches. In this paper after having proved the STI we make calculations on the equations of branches without recourse to their parametrizations. In particular we prove a new formula for the intersection multiplicity of two branches, which does not involve any reference to their parametrizations. In this way we get shorter and conceptually simpler proofs of basic theorems than in the classical approach to plane algebroid branches.

The contents of this article are

1. Preliminaries

1.1 Arithmetical lemmas and semigroups of naturals

1.2 Plane algebroid curves

2. The strong triangle inequality

3. The semigroup of a plane algebroid branch

4. A proof of the Semigroup Theorem

5. Key polynomials

6. The Abhyankar-Moh theory

7. A formula for the intersection multiplicity of two branches 
8. The Abhyankar-Moh irreducibility criterion

9. Characterization of the semigroups associated with branches

10. Description of branches with given semigroup

11. Merle-Granja's Factorization Theorem

The following notation is used in the sequel. The set of all integers (resp. non-negative integers) is denoted by $\mathbf{Z}$ (resp. $\mathbf{N}$ ). We write $\operatorname{gcd} S$ for the greatest common divisor of a nonempty subset $S \subset \mathbf{N}$. Conventions about calculating with $+\infty$ are usual. In all this note $\mathbf{K}$ is an algebraically closed field of arbitrary characteristic.

\section{Preliminaries}

In this section we fix our notations and recall some useful notions and results.

\subsection{Arithmetical lemmas and semigroups of naturals}

We recall here some properties of semigroups of natural numbers that we will use in Section 3 of this paper.

Lemma 1.1 Let $v_{0}, \ldots, v_{k}$ be a sequence of positive integers. Set $d_{i}=$ $\operatorname{gcd}\left(v_{0}, \ldots, v_{i}\right)$ for $i \in\{0,1, \ldots, k\}$ and $n_{i}=\frac{d_{i-1}}{d_{i}}$ for $i \in\{1, \ldots, k\}$. Then for every $a \in \mathbf{Z} d_{k}$ we have Bézout's relation:

$$
a=a_{0} v_{0}+a_{1} v_{1}+\cdots+a_{k} v_{k}
$$

where $a_{0} \in \mathbf{Z}$ and $0 \leq a_{i}<n_{i}$ for $i \in\{1, \ldots, k\}$. The sequence $\left(a_{0}, \ldots, a_{k}\right)$ is unique.

Proof. Existence: if $k=0$ the lemma is obvious. Suppose that $k>0$ and that the lemma is true for $k-1$. Since $\left(d_{k}\right) \mathbf{Z}=\left(d_{k-1}, v_{k}\right) \mathbf{Z}$ we can write for every $a \in\left(d_{k}\right) \mathbf{Z}: a=a^{\prime} d_{k-1}+a^{\prime \prime} v_{k}$ with $a^{\prime}, a^{\prime \prime} \in \mathbf{Z}$. For any integer $l$ we have $a=\left(a^{\prime}-l v_{k}\right) d_{k-1}+\left(a^{\prime \prime}+l d_{k-1}\right) v_{k}$. Thus we can take $a^{\prime \prime} \geq 0$. Dividing $a^{\prime \prime}$ by $\frac{d_{k-1}}{d_{k}}$ we get $a^{\prime \prime}=\left(\frac{d_{k-1}}{d_{k}}\right) a^{\prime \prime \prime}+a_{k}$ with $0 \leq a_{k}<\frac{d_{k-1}}{d_{k}}$. Therefore

$$
a=a^{\prime} d_{k-1}+\left(\frac{d_{k-1}}{d_{k}} a^{\prime \prime \prime}+a_{k}\right) v_{k}=\left(a^{\prime}+\frac{v_{k}}{d_{k}} a^{\prime \prime \prime}\right) d_{k-1}+a_{k} v_{k} .
$$


By induction hypothesis we get $\left(a^{\prime}+\frac{v_{k}}{d_{k}} a^{\prime \prime \prime}\right) d_{k-1}=a_{0} v_{0}+\cdots+a_{k-1} v_{k-1}$ with $0 \leq a_{i}<\frac{d_{i-1}}{d_{i}}$ for $0<i \leq k-1$ and we are done.

Unicity: Suppose that $a_{0} v_{0}+\cdots+a_{k} v_{k}=a_{0}^{\prime} v_{0}+\cdots+a_{k}^{\prime} v_{k}$ with $0 \leq a_{i}, a_{i}^{\prime}<$

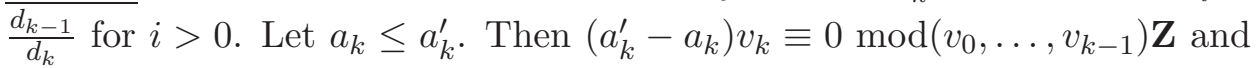
consequently $\left(a_{k}^{\prime}-a_{k}\right) v_{k} \equiv 0 \bmod \left(d_{k-1}\right) \mathbf{Z}$, which implies $\left(a_{k}^{\prime}-a_{k}\right)\left(\frac{v_{k}}{d_{k}}\right) \equiv 0$ $\bmod \frac{d_{k-1}}{d_{k}} \mathbf{Z}$.

Therefore $a_{k}^{\prime}-a_{k} \equiv 0 \bmod \frac{d_{k-1}}{d_{k}} \mathbf{Z}$ and $a_{k}^{\prime}-a_{k}=0$ since $0 \leq a_{k}^{\prime}-a_{k}<\frac{d_{k-1}}{d_{k}}$. Unicity follows by induction.

Lemma 1.2 With the above notations assume that $n_{i-1} v_{i-1}<v_{i}$ for $i \in$ $\{2, \ldots, k\}$. Then

(i) $n_{k} v_{k} \in \mathbf{N} v_{0}+\cdots+\mathbf{N} v_{k-1}$,

(ii) if $a \in \mathbf{N} v_{0}+\cdots+\mathbf{N} v_{k}$ then there are integers $a_{0}, \ldots, a_{k}$ such that $a=a_{0} v_{0}+a_{1} v_{1}+\cdots+a_{k} v_{k}$, where $0 \leq a_{0}$ and $0 \leq a_{i}<n_{i}$ for $i \in\{1, \ldots, k\}$.

\section{Proof.}

1. Since $n_{k} v_{k}=d_{k-1} \frac{v_{k}}{d_{k}} \equiv 0\left(\bmod d_{k-1} \mathbf{Z}\right)$, by Lemma 1.1 we can write Bézout's identity

$$
n_{k} v_{k}=a_{0} v_{0}+a_{1} v_{1}+\cdots+a_{k-1} v_{k-1},
$$

where $a_{0} \in \mathbf{Z}$ and $0 \leq a_{i}<n_{i}$ for $i \in\{1, \ldots, k-1\}$.

Therefore we get

$$
\begin{aligned}
a_{0} v_{0} & =n_{k} v_{k}-a_{1} v_{1}-\cdots-a_{k-1} v_{k-1} \\
& \geq n_{k} v_{k}-\left(n_{1}-1\right) v_{1}-\cdots-\left(n_{k-1}-1\right) v_{k-1} \\
& =n_{k} v_{k}-\left[\left(n_{1} v_{1}-v_{1}\right)+\cdots+\left(n_{k-1} v_{k-1}-v_{k-1}\right)\right] \\
& >n_{k} v_{k}-\left[\left(v_{2}-v_{1}\right)+\cdots+\left(v_{k}-v_{k-1}\right)\right] \\
& =n_{k} v_{k}-v_{k}+v_{1}>0
\end{aligned}
$$

which proves $(i)$. 
2. We have to check that $a \in \mathbf{N} v_{0}+\cdots+\mathbf{N} v_{k}$ implies $a_{0} \geq 0$, where $a_{0} \in \mathbf{Z}$ is defined by Bézout's identity. If $k=0$ it is obvious. Suppose that $k>0$ and that the property is true for $k-1$. By assumption we have $a=q_{0} v_{0}+\cdots+q_{k} v_{k}$ with $q_{i} \geq 0$ for $i \in\{0, \ldots, k\}$. By the Euclidean division of $q_{k}$ by $n_{k}$ we get $q_{k}=q_{k}^{\prime} n_{k}+a_{k}$ with $0 \leq a_{k}<n_{k}$. Thus $a=q_{0} v_{0}+\cdots+q_{k-1} v_{k-1}+q_{k}^{\prime} n_{k} v_{k}+a_{k} v_{k}=a^{\prime}+a_{k} v_{k}$, where $0 \leq a_{k}<n_{k}$ and $a^{\prime} \in \mathbf{N} v_{0}+\cdots+\mathbf{N} v_{k-1}$ by Property $(i)$. Use the induction hypothesis.

Remark 1.3 In fact we have proved the following property, stronger that the first part of Lemma 1.2: if $n_{k} v_{k}=a_{0} v_{0}+a_{1} v_{1}+\cdots+a_{k-1} v_{k-1}$ is Bézout's relation then $a_{0}>0$.

Remark 1.4 Obviously $n_{k} \geq 1$. From the first part of Lemma 1.2 it follows that $n_{k}>1$ if (and only if) $\mathbf{N} v_{0}+\cdots+\mathbf{N} v_{k-1} \neq \mathbf{N} v_{0}+\cdots+\mathbf{N} v_{k}$.

Let $n>0$ be an integer. A sequence of positive integers $\left(v_{0}, \ldots, v_{h}\right)$ is said to be a Seidenberg $n$-characteristic sequence or $n$-characteristic sequence if $v_{0}=n$ and it satisfies the following two axioms

1. Set $d_{i}=\operatorname{gcd}\left(v_{0}, \ldots, v_{i}\right)$ for $0 \leq i \leq h$ and $n_{i}=\frac{d_{i-1}}{d_{i}}$ for $1 \leq i \leq h$. Then $d_{h}=1$ and $n_{i}>1$ for $1 \leq i \leq h$.

2. $n_{i-1} v_{i-1}<v_{i}$ for $2 \leq i \leq h$.

Note that condition (2) implies that the sequence $\left(v_{1}, \ldots, v_{h}\right)$ is strictly increasing. If $n>1$ then $h \geq 1$. If $h=1$ then the sequence $\left(v_{0}, v_{1}\right)$ is a Seidenberg $n$-characteristic sequence if and only if $v_{0}=n$ and $\operatorname{gcd}\left(v_{0}, v_{1}\right)=$ 1. There is exactly one 1 -sequence which is (1). Note also that $2^{h} \leq n$.

If $\left(v_{0}, \ldots, v_{h}\right)$ is an $n$-characteristic sequence then for any $k \in\{1, \ldots, h\}$ the sequence $\left(\frac{v_{0}}{d_{k}}, \ldots, \frac{v_{k}}{d_{k}}\right)$ is an $\frac{n}{d_{k}}$-characteristic sequence. Its associated sequences are $\left(\frac{d_{0}}{d_{k}}, \ldots, \frac{d_{k}}{d_{k}}\right)$ and $\left(n_{1}, \ldots, n_{k}\right)$.

We say that a subset $G$ of $\mathbf{N}$ is a semigroup if it contains 0 and if it is closed under addition.

Let $G$ be a nonzero semigroup and let $n \in G, n>0$. Then there exists (cf. [He, Chapter 6, Proposition 6.1) a unique sequence $v_{0}, \ldots, v_{h}$ such that $v_{0}=n, v_{k}=\min \left(G \backslash v_{0} \mathbf{N}+\cdots+v_{k-1} \mathbf{N}\right)$ for $k \in\{1, \ldots, h\}$ and $G=$ 
$v_{0} \mathbf{N}+\cdots+v_{h} \mathbf{N}$. We call the sequence $\left(v_{0}, \ldots, v_{h}\right)$ the $n$-minimal system of generators of $G$. If $n=\min (G \backslash\{0\})$ then we say that $\left(v_{0}, \ldots, v_{h}\right)$ is the minimal set of generators of $G$. We will study semigroups generated by $n$-characteristic sequences.

Proposition 1.5 Let $G=v_{0} \mathbf{N}+\cdots+v_{h} \mathbf{N}$ where $\left(v_{0}, \ldots, v_{h}\right)$ is an $n$ characteristic sequence. Then

1. The sequence $\left(v_{0}, \ldots, v_{h}\right)$ is the $n$-minimal system of generators of $G$.

2. $\min (G \backslash\{0\})=\min \left(v_{0}, v_{1}\right)$.

3. The minimal system of generators of $G$ is $\left(v_{0}, v_{1}, \ldots, v_{h}\right)$ if $v_{0}<v_{1}$, $\left(v_{1}, v_{0}, \ldots, v_{h}\right)$ if $v_{1}<v_{0}$ and $v_{0} \not \equiv 0\left(\bmod v_{1}\right)$ and $\left(v_{1}, v_{2}, \ldots, v_{h}\right)$ if $v_{0} \equiv 0\left(\bmod v_{1}\right)$. Moreover, the minimal system of generators of $G$ is a $\min (G \backslash\{0\})$-characteristic sequence.

4. Let $c=\sum_{k=1}^{h}\left(n_{k}-1\right) v_{k}-v_{0}+1$. Then for every $a, b \in \mathbf{Z}:$ if $a+b=c-1$ then exactly one element of the pair $(a, b)$ belongs to $G$. Consequently $c$ is the smallest element of $G$ such that all integers bigger than or equal to it are in $G$.

5. $c$ is an even number and $\sharp(\mathbf{N} \backslash G)=\frac{c}{2}$.

Proof. We leave to the reader the proof of the first three claims. To prove the fourth claim (see [Sa-St] ) we take two integers $a, b \in \mathbf{Z}$ such that $a+b=c-1$. Let us write Bézout's relation $a=a_{0} v_{0}+a_{1} v_{1}+\cdots+a_{h} v_{h}$ where $a_{0} \in \mathbf{Z}$ and $0 \leq a_{i}<n_{i}$ for $i \in\{1, \ldots, h\}$. Then by definition of $c$ we get $b=c-1-a=-v_{0}+\sum_{k=1}^{h}\left(n_{k}-1\right) v_{k}-a_{0} v_{0}-\sum_{k=1}^{h} a_{k} v_{k}=-\left(a_{0}+1\right) v_{0}+$ $\sum_{k=1}^{h}\left(n_{k}-1-a_{k}\right) v_{k}$. This is a Bézout relation. To finish the proof it suffices to remark that exactly one element of the pair $\left(a_{0},-a_{0}-1\right)$ is greater than or equal to zero. For the last remark, note that $(c+N)+(-N-1)=c-1$ and hence, if $N \geq 0$, then $-N-1 \notin G$ and consequently $c+N \in G$ for all $N \geq 0$. On the other hand, since $0 \in G$ we have $c-1 \notin G$ and hence $c$ is the smallest integer such that all integers bigger than or equal to it are in $G$.

Finally we will prove the fifth claim. The mapping $[0, c-1] \cap G \ni a \rightarrow$ $c-1-a \in[0, c-1] \cap(\mathbf{N} \backslash G)$ is bijective. Therefore we have $2 \cdot \sharp([0, c-1] \cap G)=c$ and the last claim follows.

The number $c$ is called the conductor of the semigroup $G$. 


\subsection{Plane algebroid curves}

We review here some basic notions from the local theory of algebraic curves. For more details we refer the reader to [Sei2].

Let $f \in \mathbf{K}[[x, y]]$ be a non-zero power series without constant term. An algebroid curve $\{f=0\}$ is defined to be the ideal generated by $f$ in $\mathbf{K}[[x, y]]$. We say that $\{f=0\}$ is irreducible (reduced) if $f$ in $\mathbf{K}[[x, y]]$ is irreducible ( $f$ has no multiple factors). The irreducible curves are also called branches. The order ord $f$ of the power series $f$ is, by definition, the multiplicity of the curve $\{f=0\}$. The initial form in $f$ of $f$ defines the tangent lines of $\{f=0\}$. If $\{f=0\}$ is irreducible then it has only one tangent line i.e. in $f=l^{\text {ord } f}$ where $l$ is a linear form.

A formal isomorphism $\Phi$ is a pair of power series $\Phi(x, y)=(a x+b y+$ $\left.\cdots, a^{\prime} x+b^{\prime} y+\cdots\right)$ where $a b^{\prime}-a^{\prime} b \neq 0$ and the dots denote terms in $x, y$ of order bigger than 1 . The map $f \longrightarrow f \circ \Phi$ is an isomorphism of the ring $\mathbf{K}[[x, y]]$. Two curves $\{f=0\}$ and $\{g=0\}$ are said to be formally equivalent if there is a formal isomorphism $\Phi$ such that $f \circ \Phi=g \cdot$ unit.

For any power series $f, g \in \mathbf{K}[[x, y]]$ we define the intersection multiplicity or intersection number $i_{0}(f, g)$ by putting

$$
i_{0}(f, g)=\operatorname{dim}_{\mathbf{K}} \mathbf{K}[[x, y]] /(f, g),
$$

where $(f, g)$ is the ideal of $\mathbf{K}[[x, y]]$ generated by $f$ and $g$. If $f, g$ are nonzero power series without constant term then $i_{0}(f, g)<+\infty$ if and only if $\{f=0\}$ and $\{g=0\}$ have no common branch. The following properties are basic

1. if $\Phi$ is a formal isomorphism then $i_{0}(f, g)=i_{0}(f \circ \Phi, g \circ \Phi)$.

2. $i_{0}(f, g h)=i_{0}(f, g)+i_{0}(f, h)$.

Let $t$ be a variable. A parametrization is a pair $(\phi(t), \psi(t)) \in \mathbf{K}[[t]]^{2}$ such that $\phi(t) \neq 0$ or $\psi(t) \neq 0$ in $\mathbf{K}[[t]]$ and $\phi(0)=\psi(0)=0$. We say that the parametrization $(\phi(t), \psi(t))$ is good if the field of fractions of the ring $\mathbf{K}[[\phi(t), \psi(t)]]$ is equal to the field $\mathbf{K}((t))$.

Theorem 1.6 (Normalization Theorem) Let $f=f(x, y) \in \mathbf{K}[[x, y]]$ be an irreducible power series. Then there is a good parametrization $(\phi(t), \psi(t))$ such that $f(\phi(t), \psi(t))=0$. If $(\alpha(s), \beta(s)) \in \mathbf{K}[[s]]^{2}$ is a parametrization such that $f(\alpha(s), \beta(s))=0$ then there is a power series $\sigma(s) \in \mathbf{K}[[s]], \sigma(0)=$ 0 such that $\alpha(s)=\phi(\sigma(s))$ and $\beta(s)=\psi(\sigma(s))$. 
Let us recall also

Theorem 1.7 Under the above assumptions and notations, for any power series $g=g(x, y) \in \mathbf{K}[[x, y]]$ we have $i_{0}(f, g)=$ ord $g(\phi(t), \psi(t))$.

Taking $g=x$ (respect. $g=y$ ) we get from the above formula that ord $f(0, y)=$ $i_{0}(f, x)=$ ord $\phi(t)$ and ord $f(x, 0)=i_{0}(f, y)=$ ord $\psi(t)$.

Using Theorem 1.7 we check the following two properties of intersection numbers:

3. If $f$ is irreducible, then $i_{0}\left(f, g+g^{\prime}\right) \geq \inf \left\{i_{0}(f, g), i_{0}\left(f, g^{\prime}\right)\right\}$ with equality if $i_{0}(f, g) \neq i_{0}\left(f, g^{\prime}\right)$.

4. If $f$ is irreducible and $i_{0}(f, g)=i_{0}(f, h)<+\infty$ then there exists a constant $c \in \mathbf{K}$ such that $i_{0}(f, g-c h)>i_{0}(f, g)$.

In what follows we need

Lemma 1.8 Let $f(x, y) \in \mathbf{K}[[x, y]]$ be an irreducible power series such that $f(0, y) \neq 0$ and let $(\alpha(s), \beta(s)), \alpha(s) \neq 0$ in $\mathbf{K}[[s]]$, be a parametrization such that $f(\alpha(s), \beta(s))=0$. Then, for every power series $g(x, y) \in \mathbf{K}[[x, y]]$ we have

$$
\text { ord } g(\alpha(s), \beta(s))=\frac{i_{0}(f, g)}{i_{0}(f, x)} \text { ord } \alpha(s) .
$$

Proof. Let $(\phi(t), \psi(t))$ be a good parametrization of the branch $\{f(x, y)=$ $0\}$. Then $\alpha(s)=\phi(\sigma(s)), \beta(s)=\psi(\sigma(s))$ for a power series $\sigma(s) \in \mathbf{K}[[s]]$, $\sigma(0)=0$. We get ord $\alpha(s)=$ ord $\phi(t)$ ord $\sigma(s)=$ ord $f(0, y)$ ord $\sigma(s)=$ $i_{0}(f, x)$ ord $\sigma(s)$

and consequently

$$
\operatorname{ord} \sigma(s)=\frac{\operatorname{ord} \alpha(s)}{i_{0}(f, x)}
$$

On the other hand ord $g(\alpha(s), \beta(s))=$ ord $g(\phi(t), \phi(t))$.ord $\sigma(s)$ and by Theorem 1.7 we get

$$
\text { ord } g(\alpha(s), \beta(s))=i_{0}(f, g) \text { ord } \sigma(s) .
$$

Now the formula for ord $g(\alpha(s), \beta(s))$ follows.

For any irreducible power series $f \in \mathbf{K}[[x, y]]$ we put

$$
\Gamma(f)=\left\{i_{0}(f, g): g \text { runs over all power series such that } g \not \equiv 0(\bmod f)\right\} \text {. }
$$


Clearly $\Gamma(f)$ is a semigroup. We call $\Gamma(f)$ the semigroup associated with the branch $\{f=0\}$.

Two branches $\{f=0\}$ and $\{g=0\}$ are equisingular if and only if $\Gamma(f)=$ $\Gamma(g)$. Two formally equivalent branches are equisingular. The branch $\{f=$ $0\}$ is non-singular (that is of multiplicity 1 ) if and only if $\Gamma(f)=\mathbf{N}$. We have $\min (\Gamma(f) \backslash\{0\})=$ ord $f$.

Different (but equivalent) definitions of equisingularity were given by Zariski in [a1].

Note that the mapping $g \mapsto i_{0}(f, g)$ induces a valuation $v_{f}$ of the ring $\mathbf{K}[[x, y]] /(f)$. The semigroup $\Gamma(f)$ can be described as the semigroup of values of $v_{f}$.

\section{The strong triangle inequality}

In this section we generalize the well-known property of the intersection multiplicity $[\mathrm{Pt}]$ to any characteristic. Let us begin with the notion of logarithmic distance.

Let $A$ be a non-empty set. A function $d: A \times A \longrightarrow \mathbf{R} \cup\{+\infty\}$ satisfying for arbitrary $a, b, c \in A$, the conditions:

(i) $d(a, a)=+\infty$,

(ii) $d(a, b)=d(b, a)$,

(iii) $d(a, b) \geq \inf \{d(a, c), d(b, c)\}$,

will be called a logarithmic distance (for short log-distance). We call the third property the Strong Triangle Inequality (the STI). It is equivalent to the following

(iii') at least two of the numbers $d(a, b), d(a, c), d(b, c)$ are equal and the third is not smaller than the other two.

Lemma 2.1 Let $d$ be a log-distance in the set $A$. For any $a_{1}, \ldots, a_{m}$, $b_{1}, \ldots, b_{n}, c \in A$ at least one of the following conditions holds:

(I) there exists a $j \in\{1, \ldots, n\}$ such that for any $i \in\{1, \ldots, m\}, d\left(a_{i}, c\right) \leq$ $d\left(a_{i}, b_{j}\right)$,

(II) there exists an $i \in\{1, \ldots, m\}$ such that for any $j \in\{1, \ldots, n\}, d\left(b_{j}, c\right) \leq$ $d\left(a_{i}, b_{j}\right)$. 
Proof. Let us suppose that neither (I) nor (II) holds. Then, for any $j \in$ $\{1, \ldots, n\}$ there exists an index $p(j) \in\{1, \ldots, m\}$ such that $d\left(a_{p(j)}, c\right)>$ $d\left(a_{p(j)}, b_{j}\right)$ and, for any $i \in\{1, \ldots, m\}$, there exists $s(i) \in\{1, \ldots, n\}$ such that $d\left(b_{s(i)}, c\right)>d\left(a_{i}, b_{s(i)}\right)$. Applying the STI to $a_{p(j)}, b_{j}, c$ and to $a_{i}, b_{s(i)}, c$ we get

$$
d\left(a_{p(j)}, b_{j}\right)=d\left(b_{j}, c\right)<d\left(a_{p(j)}, c\right),
$$

and

$$
d\left(a_{i}, b_{s(i)}\right)=d\left(a_{i}, c\right)<d\left(b_{s(i)}, c\right) .
$$

We may assume without loss of generality that

$$
d\left(a_{p(1)}, b_{1}\right)=\sup _{j=1}^{n}\left\{d\left(a_{p(j)}, b_{j}\right)\right\} .
$$

Using successively (1), (2) and again (1), we get

$$
d\left(a_{p(1)}, b_{1}\right)<d\left(a_{p(1)}, c\right)=d\left(a_{p(1)}, b_{s(p(1))}\right)<d\left(b_{s(p(1))}, c\right)=d\left(a_{p\left(j_{1}\right)}, b_{j_{1}}\right)
$$

with $j_{1}=s(p(1))$. Thus we have $d\left(a_{p(1)}, b_{1}\right)<d\left(a_{p\left(j_{1}\right)}, b_{j_{1}}\right)$, which contradicts assumption (3).

An important log-distance on the set of branches can be defined by means of the intersection multiplicity. Let $\{l=0\}$ be a smooth branch. For any branches $\{f=0\}$ and $\{g=0\}$ different from the branch $\{l=0\}$ we put

$$
d_{l}(f, g)=\frac{i_{0}(f, g)}{i_{0}(f, l) i_{0}(g, l)} .
$$

Our aim is to prove

Theorem 2.2 The function $d_{l}$ is a log-distance in the set of all branches different from $\{l=0\}$.

Proof. (cf. Chad-Pt])

We may assume $l=x$. Since $d_{x}(f, f)=+\infty$ and $d_{x}(f, g)=d_{x}(g, f)$ it suffices to check the STI. Let $\{f=0\},\{g=0\}$ and $\{h=0\}$ be three branches different from $\{x=0\}$. Let $m=i_{0}(f, x)=$ ord $f(0, y), n=i_{0}(g, x)=$ ord $g(0, y), p=i_{0}(h, x)=$ ord $h(0, y)$. Using the Weierstrass preparation theorem we may assume that $f, g, h$ are distinguished polynomials of degree $m, n, p$ respectively. Using the Normalization Theorem we check (see [Sei2], Theorem 21.18) that there exist power series $\alpha(s), \alpha_{i}(s), \beta_{j}(s)$ and $\gamma_{k}(s)$ 
such that $f(\alpha(s), y)=\prod_{i=1}^{m}\left(y-\alpha_{i}(s)\right), g(\alpha(s), y)=\prod_{j=1}^{n}\left(y-\beta_{j}(s)\right)$ and $h(\alpha(s), y)=\prod_{k=1}^{p}\left(y-\gamma_{k}(s)\right)$.

The function $d: \mathbf{K}[[s]] \times \mathbf{K}[[s]] \longrightarrow \mathbf{R} \cup\{+\infty\}$ given by $d(\alpha(s), \beta(s))=$ $\operatorname{ord}(\alpha(s)-\beta(s))$ is a log-distance in $\mathbf{K}[[s]]$. Fix $k \in\{1, \ldots, p\}$ and use Lemma 2.1 to $\alpha_{1}(s), \ldots, \alpha_{m}(s), \beta_{1}(s), \ldots, \beta_{n}(s)$ and $\gamma(s)=\gamma_{k}(s)$. Then

(I) there exists a $j \in\{1, \ldots, n\}$ such that ord $\left(\alpha_{i}(s)-\gamma(s)\right) \leq$ ord $\left(\alpha_{i}(s)-\right.$ $\left.\beta_{j}(s)\right)$ for all $i \in\{1, \ldots, m\}$, or

(II) there exists an $i \in\{1, \ldots, m\}$ such that ord $\left(\beta_{j}(s)-\gamma(s)\right) \leq$ ord $\left(\alpha_{i}(s)-\right.$ $\left.\beta_{j}(s)\right)$, for all $j \in\{1, \ldots, n\}$.

If (I) holds then $\sum_{i=1}^{m}$ ord $\left(\alpha_{i}(s)-\gamma(s)\right) \leq \sum_{i=1}^{m}$ ord $\left(\alpha_{i}(s)-\beta_{j}(s)\right)$ that is ord $f(\alpha(s), \gamma(s)) \leq$ ord $f\left(\alpha(s), \beta_{j}(s)\right)$. By Lemma1.8 we get $\frac{i_{0}(h, f)}{i_{0}(x, h)} \leq \frac{i_{0}(g, f)}{i_{0}(x, g)}$ which implies $d_{l}(f, h) \leq d_{l}(f, g)$.

If (II) holds then $\sum_{j=1}^{n}$ ord $\left(\beta_{j}(s)-\gamma(s)\right) \leq \sum_{j=1}^{n}$ ord $\left(\alpha_{i}(s)-\beta_{j}(s)\right)$ that is ord $g(\alpha(s), \gamma(s)) \leq$ ord $g\left(\alpha(s), \alpha_{i}(s)\right)$ and again by Lemma 1.8 we get $\frac{i_{0}(h, g)}{i_{0}(x, h)} \leq \frac{i_{0}(f, g)}{i_{0}(x, f)}$ which implies $d_{l}(g, h) \leq d_{l}(f, g)$.

Consequently $d_{l}(f, g) \geq \inf \left\{d_{l}(f, h), d_{l}(g, h)\right\}$.

Corollary 2.3 The function $d(f, g)=\frac{i_{0}(f, g)}{\operatorname{ord} f \text { ord } g}$ is a log-distance in the set of all branches.

\section{The semigroup of a plane algebroid branch}

The aim of this section is to study the structure of the semigroup associated with a plane branch. We follow the method developped by Seidenberg in Sei1.

Let $f=f(x, y) \in \mathbf{K}[[x, y]]$ be an irreducible power series and let $\Gamma(f)$ be the semigroup associated with the branch $\{f=0\}$. Suppose that $\{f=0\} \neq$ $\{x=0\}$ and put $n=i_{0}(f, x)$. Let $\left(\overline{b_{0}}, \ldots, \overline{b_{h}}\right), \overline{b_{0}}=n$ be the $n$-minimal system of generators of $\Gamma(f)$.

Lemma 3.1 $\Gamma(f)$ is a numerical semigroup i.e. $\operatorname{gcd}(\Gamma(f))=1$.

Proof. Let $(\phi(t), \psi(t))$ be a good parametrization of the branch $f(x, y)=0$. Then we have $\mathbf{K}((t))=\mathbf{K}((\phi(t), \psi(t)))$ and we can write $t=\frac{p(\phi(t), \psi(t))}{q(\phi(t), \psi(t))}$ 
for some $p(x, y), q(x, y) \in \mathbf{K}[[x, y]], q \not \equiv 0(\bmod f)$. Taking orders gives $1=i_{0}(f, p)-i_{0}(f, q)$. Put $a:=i_{0}(f, p)$ and $b:=i_{0}(f, q)$. Then $a, b \in \Gamma(f)$ and $\operatorname{gcd}(a, b)=1$, which proves the lemma.

We put $e_{0}=n, e_{k}=\operatorname{gcd}\left(e_{k-1}, \overline{b_{k}}\right)$ for $k \in\{1, \ldots, h\}$ and $n_{k}=\frac{e_{k-1}}{e_{k}}$ for $k \in\{1, \ldots, h\}$. By Lemma 3.1 we have $e_{h}=1$. In what follows we write $v_{f}(g)$ instead of $i_{0}(f, g)$.

Theorem 3.2 (Semigroup Theorem) Let $\{f=0\}$ be a branch such that $\{f=0\} \neq\{x=0\}$. Set $n=v_{f}(x)$ and let $\overline{b_{0}}, \ldots, \overline{b_{h}}$ be the $n$-minimal system of generators of the semigroup $\Gamma(f)$. There exists a sequence of monic polynomials $f_{0}, f_{1}, \ldots, f_{h-1} \in \mathbf{K}[[x]][y]$ such that for $k \in\{1, \ldots, h\}$ :

$\left(a_{k}\right) \operatorname{deg}_{y}\left(f_{k-1}\right)=\frac{n}{e_{k-1}}$,

$\left(b_{k}\right) v_{f}\left(f_{k-1}\right)=\overline{b_{k}}$ for $k \in\{1, \ldots, h\}$,

$\left(c_{k}\right)$ if $k>1$ then $n_{k-1} \overline{b_{k-1}}<\overline{b_{k}}$.

Moreover $n_{k}>1$ for all $k \in\{1, \ldots, h\}$.

We give the proof of the Semigroup Theorem in Section 4. The sequence $\overline{b_{0}}, \ldots, \overline{b_{h}}$ is a Seidenberg $n$-characteristic sequence and will be called the Seidenberg $n$-characteristic of the branch $\{f=0\}$ (with respect to the regular branch $\{x=0\})$. We will write $\overline{\operatorname{char}_{x}} f=\left(\overline{b_{0}}, \ldots, \overline{b_{h}}\right)$. Therefore $\overline{\operatorname{char}_{x}} f$ is determined by $n=v_{f}(x)$ and the semigroup $\Gamma(f)$. Let $f_{h}$ be the distinguished polynomial associated with $f$ and let $\overline{b_{h+1}}=+\infty$. Then $\operatorname{deg}_{y} f_{h}=\frac{n}{e_{h}}=n$ and $v_{f}\left(f_{h}\right)=\overline{b_{h+1}}=+\infty$. The polynomials $f_{0}, f_{1}, \ldots, f_{h} \in \mathbf{K}[[x]][y]$ will be called key polynomials of $f$. They are not uniquely determined by $f$.

Corollary 3.3 Suppose that two branches $\{f=0\}$ and $\{g=0\}$ intersect the axis $\{x=0\}$ with the same multiplicity $n<+\infty$. Then $\overline{\operatorname{char}}_{x} f=\overline{\operatorname{char}}_{x} g$ if and only if $\{f=0\}$ and $\{g=0\}$ are equisingular.

Let $\overline{\beta_{0}}, \ldots, \overline{\beta_{g}}$ be the minimal system of generators of the semigroup $\Gamma(f)$ $\left(\overline{\beta_{0}}=\min \{\Gamma(f) \backslash\{0\}\}=\right.$ ord $\left.f\right)$. We put $\overline{\operatorname{char}} f=\left(\overline{\beta_{0}}, \ldots, \overline{\beta_{g}}\right)$. Note that $\overline{\operatorname{char}} f=\overline{\operatorname{char}}_{x} f$ if and only if $v_{f}(x)=$ ord $f$.

Corollary 3.4 (Inversion formulae) Let $\overline{\operatorname{char}}_{x} f=\left(\overline{b_{0}}, \overline{b_{1}}, \ldots, \overline{b_{h}}\right)$. Then $\overline{\operatorname{char}} f=\overline{\operatorname{char}_{x}} f$ if and only if $\overline{b_{0}}<\overline{b_{1}}$. If $\overline{b_{1}}<\overline{b_{0}}$ and $\overline{b_{0}} \neq 0\left(\bmod \overline{b_{1}}\right)$ then $\overline{\operatorname{char}} f=\left(\overline{b_{1}}, \overline{b_{0}}, \ldots, \overline{b_{h}}\right)$. If $\overline{b_{0}} \equiv 0\left(\bmod \overline{b_{1}}\right)$ then $\overline{\operatorname{char}} f=\left(\overline{b_{1}}, \overline{b_{2}}, \ldots, \overline{b_{h}}\right)$. 
Proof. The corollary follows from the Semigroup Theorem and from the third claim of Proposition 1.5.

Let $\overline{\mathcal{O}}$ be the normalization of the $\operatorname{ring} \mathcal{O}=\mathbf{K}[[x, y]] /(f)$ and let $\mathcal{C}$ be the conductor ideal of $\overline{\mathcal{O}}$ in $\mathcal{O}$. Put $c(f)=\operatorname{dim}_{\mathbf{K}} \overline{\mathcal{O}} / \mathcal{C}$. Then $c(f)$ is the smallest element of $\Gamma(f)$ such that $c(f)+N \in \Gamma(f)$ for any integer $N \geq 0$ (see Cam1, p. 136).

Corollary 3.5 (Conductor formula) If $\overline{\operatorname{char}}_{x} f=\left(\overline{b_{0}}, \overline{b_{1}}, \ldots, \overline{b_{h}}\right)$ then $c(f)=\sum_{k=1}^{h}\left(n_{k}-1\right) \overline{b_{k}}-\overline{b_{0}}+1$.

Proof. Use the Semigroup Theorem and the fourth claim of Proposition 1.5.

\section{Notes}

Seidenberg gave in Sei1 the description of the semigroup of a zero-dimensional valuation of the extension $\mathbf{K}(x, y) / \mathbf{K}$ ([Sei1], Theorem 6, p. 398) in terms of generators. The case of the semigroup associated with an algebroid plane branch was studied by Azevedo in $\mathrm{Az}$. His method based on the Apèry sequences was extended by Angermüller in Ang to the case of arbitrary characteristic. For different characterizations of the numerical semigroups we refer the reader to $\mathrm{He}$, Chapter 6.

If $n=v_{f}(x) \not \equiv 0(\bmod$ char $\mathbf{K})$ the Puiseux series are available. Zariski in Za3] (see also $\mathrm{Gw}-\mathrm{P} 1],[\mathrm{Po}]$ ) constructed the sequence $\overline{\beta_{0}}, \ldots, \overline{\beta_{g}}$ and the corresponding sequence of key polynomials by using Puiseux series expansion determined by the equation $f(x, y)=0$. This method turned out efficient when applied to the semigroups of integers associated with meromorphic curves (see [A-M1, A3]). A proof of the Semigroup Theorem based on the Hamburger-Noether expansion was given by Russel in [Ru] and Campillo in Cam1, Cam2. To describe classes of equisingular plane algebroid branches one uses characteristic pairs (see [Moh1], [LJ]).

\section{A proof of the Semigroup Theorem}

Let $\{f=0\}$ be a branch such that $n=i_{0}(f, x)<+\infty$ and let $\overline{b_{0}}, \ldots, \overline{b_{h}}$ be the $n$-minimal system of generators of the semigroup $\Gamma(f)$. Observe that by the Weierstrass Division Theorem:

$$
\Gamma(f)=\left\{v_{f}(g): g \in \mathbf{K}[[x]][y] \backslash\{0\}: \operatorname{deg}_{y} g<n\right\} .
$$

Proposition 4.1 There exists a monic polynomial $f_{0} \in \mathbf{K}[[x]][y]$ such that 
$\left(a_{1}\right) \operatorname{deg}_{y}\left(f_{0}\right)=\frac{n}{e_{0}}=1$,

$\left(b_{1}\right) v_{f}\left(f_{0}\right)=\overline{b_{1}}$.

To prove Proposition 4.1 we check the following three properties:

Lemma 4.2 (Property $\mathbf{I}_{0}$ ) If $\psi$ is a non-zero polynomial with $\operatorname{deg}_{y} \psi<1$ then $v_{f}(\psi) \in \mathbf{N} \overline{b_{0}}$.

Proof. Obviously $\psi \in \mathbf{K}[[x]]$. Thus $v_{f}(\psi)=($ ord $\psi) v_{f}(x) \in \mathbf{N} \overline{b_{0}}$.

Lemma 4.3 (Property $\left.\mathbf{I I}_{0}\right)$ If $\operatorname{deg}_{y} \psi<1$ then $v_{f}(y+\psi) \leq \overline{b_{1}}$.

Proof. Let $g \in \mathbf{K}[[x]][y]$ be such that $v_{f}(g)=\overline{b_{1}}$. By the Euclidean division we get $g=Q \cdot(y+\psi)+\psi_{1}$ with $\psi_{1} \in \mathbf{K}[[x]]$. Clearly $v_{f}(g) \neq v_{f}\left(\psi_{1}\right)$ and we get $\overline{b_{1}} \geq \inf \left\{v_{f}(g), v_{f}\left(\psi_{1}\right)\right\}=v_{f}\left(g-\psi_{1}\right)=v_{f}(Q \cdot(y+\psi)) \geq v_{f}(y+\psi)$.

Lemma 4.4 (Property $\left.\mathbf{I I I}_{0}\right)$ If $\psi \in \mathbf{K}[[x]]$ and $v_{f}(y+\psi) \in \mathbf{N} \overline{b_{0}}$ then there exists a power series $\bar{\psi} \in \mathbf{K}[[x]]$ such that $v_{f}(y+\bar{\psi})>v_{f}(y+\psi)$.

Proof. There exists an integer $a \geq 0$ such that $v_{f}(y+\psi)=a \overline{b_{0}}=v_{f}\left(x^{a}\right)$. Therefore there is an element $c \in \mathbf{K}$ such that $v_{f}\left(y+\psi-c x^{a}\right)>v_{f}(y+\psi)$. We put $\bar{\psi}=\psi-c x^{a}$.

Proof of Proposition 4.1. From Properties $\left(\mathrm{II}_{0}\right)$ and $\left(\mathrm{III}_{0}\right)$ it follows that there exists a monic polynomial $f_{0}$ of degree 1 such that $v_{f}\left(f_{0}\right) \notin \mathbf{N} \overline{b_{0}}$. By definition of $\overline{b_{1}}$ we get $v_{f}\left(f_{0}\right) \geq \overline{b_{1}}$. The equality follows from Property $\left(\mathrm{II}_{0}\right)$.

Proposition 4.5 Suppose that there exist monic polynomials $f_{0}, f_{1}, \ldots, f_{k-1}$ in $\mathbf{K}[[x]][y]$ such that

$\left(a_{i}\right) \operatorname{deg}_{y}\left(f_{i-1}\right)=\frac{n}{e_{i-1}}$

(bi $) v_{f}\left(f_{i-1}\right)=\overline{b_{i}}$ for $i \in\{1, \ldots, k\}$,

(ci) $n_{i-1} \overline{b_{i-1}}<\overline{b_{i}}$ for $i \in\{2, \ldots, k\}$.

Then there exists a monic polynomial $f_{k} \in \mathbf{K}[[x]][y]$ such that

$$
\begin{aligned}
& \left(a_{k+1}\right) \operatorname{deg}_{y}\left(f_{k}\right)=\frac{n}{e_{k}}, \\
& \left(b_{k+1}\right) v_{f}\left(f_{k}\right)=\overline{b_{k+1}},
\end{aligned}
$$


$\left(c_{k+1}\right) n_{k} \overline{b_{k}}<\overline{b_{k+1}}$.

To prove Proposition 4.5 we check the following three properties:

Lemma 4.6 (Property $\mathbf{I}_{k}$ ) If $\psi$ is a non-zero polynomial with $\operatorname{deg}_{y} \psi<$ $\frac{n}{e_{k}}$ then $v_{f}(\psi) \in \mathbf{N} \overline{b_{0}}+\cdots+\mathbf{N} \overline{b_{k}}$.

Proof. Let $l \leq k$. We will prove that for $\operatorname{deg}_{y} \psi<\frac{n}{e_{l}}$ we have $v_{f}(\psi) \in$ $\mathbf{N} \overline{b_{0}}+\cdots+\mathbf{N} \overline{b_{l}}$. We proceed by induction on $l$. The case $l=0$ is already proved (see Property $\mathrm{I}_{0}$ ). Let $l>0$ and suppose the property holds for polynomials of degree less than $\frac{n}{e_{l-1}}$. Fix $\psi \in \mathbf{K}[[x]][y]$ with $\operatorname{deg}_{y}(\psi)<\frac{n}{e_{l}}$ and consider the $f_{l-1}$-adic expansion of $\psi$ :

$$
\psi=\psi_{0} f_{l-1}^{s}+\psi_{1} f_{l-1}^{s-1}+\cdots+\psi_{s},
$$

where $\psi_{0} \neq 0, \operatorname{deg}_{y}\left(\psi_{i}\right)<\operatorname{deg}_{y}\left(f_{l-1}\right)=\frac{n}{e_{l-1}}$.

Note that $s \leq \frac{\operatorname{deg}_{y}(\psi)}{\operatorname{deg}_{y}\left(f_{l-1}\right)}<n_{l}$. Let $I$ be the set of all $i \in\{0, \ldots, s\}$ such that $\psi_{i} \neq 0$. Therefore, by the induction hypothesis we get $v_{f}\left(\psi_{i}\right) \in \mathbf{N} \overline{b_{0}}+$ $\cdots+\mathbf{N} \overline{b_{l-1}}$, and

$$
v_{f}\left(\psi_{i}\right) \equiv 0 \bmod e_{l-1} \text { for } i \in I \text {. }
$$

Moreover

$$
v_{f}\left(\psi_{i} f_{l-1}^{s-i}\right) \neq v_{f}\left(\psi_{j} f_{l-1}^{s-j}\right) \text { for } i \neq j \in I .
$$

Indeed, suppose that (6) is not true, so there exist $i, j \in I$ such that $i<j$ and $v_{f}\left(\psi_{i} f_{l-1}^{s-i}\right)=v_{f}\left(\psi_{j} f_{l-1}^{s-j}\right)$. Therefore $v_{f}\left(\psi_{i}\right)+(s-i) v_{f}\left(f_{l-1}\right)=v_{f}\left(\psi_{j}\right)+$ $(s-j) v_{f}\left(f_{l-1}\right)$ and $(j-i) \overline{b_{l}}=v_{f}\left(\psi_{j}\right)-v_{f}\left(\psi_{i}\right) \equiv 0 \bmod e_{l-1}$ by (15). The last relation implies $(j-i) \frac{\overline{b_{l}}}{e_{l}} \equiv 0 \bmod n_{l}$ and consequently $j-i \equiv 0$ $\bmod n_{l}$ because $\frac{\overline{b_{l}}}{e_{l}}$ and $n_{l}$ are co-prime. We get a contradiction because $0<j-i \leq s<n_{l}$. Now by (4) and (6) we get

$$
\begin{aligned}
v_{f}(\psi) & =\min _{i=0}^{s} v_{f}\left(\psi_{i} f_{l-1}^{s-i}\right)=v_{f}\left(\psi_{j} f_{l-1}^{s-j}\right) \\
& =v_{f}\left(\psi_{j}\right)+(s-j) \overline{b_{l}} \in \mathbf{N} \overline{b_{0}}+\cdots+\mathbf{N} \overline{b_{l}}
\end{aligned}
$$

for some $j \in I$.

Lemma 4.7 (Property $\left.\mathbf{I I}_{k}\right)$ If $\operatorname{deg}_{y} \psi<\frac{n}{e_{k}}$ then $v_{f}\left(y^{\frac{n}{e_{k}}}+\psi\right) \leq \overline{b_{k+1}}$. 
Proof. Let $g \in \mathbf{K}[[x]][y]$ be such that $v_{f}(g)=\overline{b_{k+1}}$. By the Euclidean division we get $g=Q \cdot\left(y^{\frac{n}{e_{k}}}+\psi\right)+\psi_{1}$ with $\psi_{1} \in \mathbf{K}[[x]][y]$ and $\operatorname{deg}_{y} \psi_{1}<\frac{n}{e_{k}}$. We may assume $\psi_{1} \neq 0$. Therefore $v_{f}\left(\psi_{1}\right) \in \mathbf{N} \overline{b_{0}}+\cdots+\mathbf{N} \overline{b_{k}}$ by Property $\left(\mathrm{I}_{k}\right)$ and $v_{f}(g)=\overline{b_{k+1}} \neq v_{f}\left(\psi_{1}\right)$. Now we get $\overline{b_{k+1}} \geq \inf \left\{v_{f}(g), v_{f}\left(\psi_{1}\right)\right\}=$ $v_{f}\left(g-\psi_{1}\right)=v_{f}\left(Q \cdot\left(y^{\frac{n}{e_{k}}}+\psi\right)\right) \geq v_{f}\left(y^{\frac{n}{e_{k}}}+\psi\right)$.

Lemma 4.8 (Property $\left.\mathbf{I I I}_{k}\right)$ If $\psi \in \mathbf{K}[[x]][y]$ with $\operatorname{deg}_{y} \psi<\frac{n}{e_{k}}$ and $v_{f}\left(y^{\frac{n}{e_{k}}}+\right.$ $\psi) \in \mathbf{N} \overline{b_{0}}+\cdots+\mathbf{N} \overline{b_{k}}$ then there is a polynomial $\bar{\psi} \in \mathbf{K}[[x]][y]$, $\operatorname{deg}_{y} \bar{\psi}<\frac{n}{e_{k}}$ such that $v_{f}\left(y^{\frac{n}{e_{k}}}+\bar{\psi}\right)>v_{f}\left(y^{\frac{n}{e_{k}}}+\psi\right)$.

Proof. By Lemma 1.2 any element of the semigroup $\mathbf{N} \overline{b_{0}}+\cdots+\mathbf{N} \overline{b_{k}}$ has the form $a_{0} \overline{b_{0}}+a_{1} \overline{b_{1}}+\cdots+a_{k} \overline{b_{k}}$ with $a_{0} \geq 0$ and $0 \leq a_{i}<n_{i}$ for $i \in\{1, \ldots, k\}$. Therefore we can write $v_{f}\left(y^{\frac{n}{e_{k}}}+\psi\right)=v_{f}\left(x^{a_{0}} f_{0}^{a_{1}} \cdots f_{k-1}^{a_{k}}\right)$ and there is an element $c \in \mathbf{K}$ such that $v_{f}\left(y^{\frac{n}{e_{k}}}+\psi-c x^{a_{0}} f_{0}^{a_{1}} \cdots f_{k-1}^{a_{k}}\right)>v_{f}\left(y^{\frac{n}{e_{k}}}+\psi\right)$. Let $\bar{\psi}=\psi-c x^{a_{0}} f_{0}^{a_{1}} \cdots f_{k-1}^{a_{k}}$. Then we have $v_{f}\left(y^{\frac{n}{e_{k}}}+\bar{\psi}\right)>v_{f}\left(y^{\frac{n}{e_{k}}}+\psi\right)$. Since $\operatorname{deg}_{y}\left(x^{a_{0}} f_{0}^{a_{1}} \cdots f_{k-1}^{a_{k}}\right)=a_{1}+a_{2} \frac{n}{e_{1}}+\cdots+\left(a_{k}\right) \frac{n}{e_{k-1}} \leq\left(n_{1}-1\right)+\left(n_{2}-1\right) \frac{n}{e_{1}}+$ $\cdots+\left(n_{k}-1\right) \frac{n}{e_{k-1}}=\frac{n n_{k}}{e_{k-1}}-1<\frac{n}{e_{k}}, \operatorname{deg}_{y} \bar{\psi}<\frac{n}{e_{k}}$.

Proof of Proposition 4.5. From Properties $\left(\mathrm{II}_{k}\right)$ and $\left(\mathrm{III}_{k}\right)$ it follows that there exists a monic polynomial $f_{k}$ of degree $\frac{n}{e_{k}}$ such that $v_{f}\left(f_{k}\right) \notin$ $\mathbf{N} \overline{b_{0}}+\cdots+\mathbf{N} \overline{b_{k}}$. By definition of $\overline{b_{k+1}}$ we get $v_{f}\left(f_{k}\right) \geq \overline{b_{k+1}}$. The equality follows from Property $\left(\mathrm{II}_{k}\right)$.

To check $\left(c_{k+1}\right)$ observe that $v_{f}\left(f_{k-1}^{n_{k}}\right)=n_{k} \overline{b_{k}}$ and $\operatorname{deg}_{y} f_{k-1}^{n_{k}}=n_{k} \frac{n}{e_{k-1}}=\frac{n}{e_{k}}$. Therefore $n_{k} \overline{b_{k}} \leq \overline{b_{k+1}}$ by Property $\left(\mathrm{II}_{k}\right)$. By Lemma 1.2 we get $n_{k} \overline{b_{k}}<\overline{b_{k+1}}$ since $\overline{b_{k+1}} \notin \mathbf{N} \overline{b_{0}}+\cdots+\mathbf{N} \overline{b_{k}}$.

Proof of Theorem 3.2. The theorem follows by induction from Proposition 4.1. Proposition 4.5 and from Remark 1.4.

Remark 4.9 From Theorem 3.2 and Lemmas 4.6, 4.7, and 4.8 it follows that Properties $I_{k}, I I_{k}$ and $I I I_{k}$ hold for all $0 \leq k<h$.

Remark 4.10 Let $\{f=0\} \neq\{x=0\}$ be a branch such that $n=i_{0}(f, x)>$ 1. Let $\overline{b_{0}}, \ldots, \overline{b_{h}}, \overline{b_{0}}=n$ be the $n$-minimal system of generators of $\Gamma(f)$. Suppose that the first $k+1$ terms $\overline{b_{0}}, \ldots, \overline{b_{k}}$, with $k<h$ are given. Let $f_{k} \in \mathbf{K}[[x]][y]$ be a monic polynomial of degree $\frac{n}{e_{k}}$ such that $i_{0}\left(f, f_{k}\right) \not \equiv 0$ $\left(\bmod e_{k}\right)$. Then by Property $I I_{k}$ we get $i_{0}\left(f, f_{k}\right) \leq \overline{b_{k+1}}$. The inequality $i_{0}\left(f, f_{k}\right) \geq \overline{b_{k+1}}$ follows from the fact that $i_{0}\left(f, f_{k}\right) \notin \mathbf{N} \overline{b_{0}}+\cdots+\mathbf{N} \overline{b_{k}}$. We get $i_{0}\left(f, f_{k}\right)=\overline{b_{k+1}}$ and $f_{k}$ is a $k$-th key polynomial of $f$. 


\section{Examples 4.11}

Let $\mathbf{K}$ be an algebraically closed field of characteristic $p>2$.

A. Let $f(x, y)=y^{p^{2}-1}+y^{p}-x^{p^{2}}=\left(y-x^{p}\right)^{p}+y^{p^{2}-1}$. Put $(\phi(t), \psi(t))=$ $\left(t^{p}+t^{p^{2}-1}, t^{p^{2}}\right)$. We have $f(\phi(t), \psi(t))=0$ and $f$ is irreducible in $\mathbf{K}[[x, y]]$. Since the order of $f$ is the prime number $p$ we get $\overline{\operatorname{char}} f=$ $\left(\overline{b_{0}}, \overline{b_{1}}\right)$ where $\overline{b_{0}}=$ ord $f=p$. To calculate $\overline{b_{1}}$ we have to construct a monic polynomial $f_{0}=y+\cdots$ of degree 1 such that $i_{0}\left(f, f_{0}\right) \not \equiv 0$ $\left(\bmod e_{0}\right), e_{0}=\overline{b_{0}}=p$. Take $g=y-x^{p}$. Then $i_{0}(f, g)=p\left(p^{2}-1\right)=$ $i_{0}\left(f, x^{p^{2}-1}\right)$. There is a constant $c \in \mathbf{K}$ such that $i_{0}\left(f, g-c x^{p^{2}-1}\right)>$ $i_{0}(f, g)$. Using the parametrization $(\phi(t), \psi(t))$ we find that $c=-1$. Let $f_{0}=g-c x^{p^{2}-1}=y-x^{p}+x^{p^{2}-1}$. Then $i_{0}\left(f, f_{0}\right)=p^{3}+p^{2}-2 p-1 \not \equiv$ $0(\bmod p)$ and by Remark 4.10 we get $\overline{b_{1}}=p^{3}+p^{2}-2 p-1$ and consequently $\overline{\operatorname{char}} f=\left(p, p^{3}+p^{2}-2 p-1\right)$.

B. Let $f(x, y)=y^{p^{2}-1}+y^{p^{2}-p}-x^{p^{2}}=\left(y^{p-1}-x^{p}\right)^{p}+y^{p^{2}-1}$ and $(\phi(t), \psi(t))=$ $\left(t^{p^{2}-p}+t^{p^{2}-1}, t^{p^{2}}\right)$. We have $f(\phi(t), \psi(t))=0$ and $f$ is irreducible in $\mathbf{K}[[x, y]]$. Since ord $f=p^{2}-p$ and $i_{0}(f, y)=p^{2} \not \equiv 0\left(\bmod \left(p^{2}-p\right)\right)$ we get $\overline{b_{0}}=p^{2}-p, \overline{b_{1}}=p^{2}, e_{1}=\operatorname{gcd}\left(\overline{b_{0}}, \overline{b_{1}}\right)=p$ and $\overline{\operatorname{char}} f=\left(\overline{b_{0}}, \overline{b_{1}}, \overline{b_{2}}\right)$. To compute $\overline{b_{2}}$ we have to construct a monic polynomial $f_{1} \in \mathbf{K}[[x]][y]$ of degree $\frac{\overline{b_{0}}}{e_{1}}=p-1$ such that $i_{0}\left(f, f_{1}\right) \not \equiv 0(\bmod p)$. Starting with the polynomial $g=y^{p-1}-x^{p}$ and proceeding like in Example $A$ we find $f_{1}=y^{p-1}-x^{p}+x^{p+1}$. Since $i_{0}\left(f, f_{1}\right)=p^{3}-1$ we get $\overline{b_{2}}=p^{3}-1$ and consequently $\overline{\operatorname{char}} f=\left(p^{2}-p, p^{2}, p^{3}-1\right)$.

\section{Key polynomials}

The key polynomials under the name of semi-roots were studied by Abhyankar [A2] and Popescu-Pampu [Po]. Here we propose the treatment without any restriction on the field characteristic.

Let $f=f(x, y) \in \mathbf{K}[[x, y]]$ be an irreducible power series such that $i_{0}(f, x)=$ ord $f(0, y)=n<+\infty$ and let $\overline{\operatorname{char}}_{x} f=\left(\overline{b_{0}}, \ldots, \overline{b_{h}}\right), \overline{b_{0}}=n$. Let $k \in$ $\{0, \ldots, h\}$. Recall that a monic polynomial $g \in \mathbf{K}[[x]][y]$ is a $k$-th key polynomial of $f$ if $\operatorname{deg}_{y} g=\frac{n}{e_{k}}$ and $v_{f}(g)=\overline{b_{k+1}}$. By the Semigroup Theorem, for any $k \in\{0, \ldots, h\}$ there exists a $k$-th key polynomial of $f$. We fix a sequence $f_{0}, \ldots, f_{h}$ of key polynomials of $f$ such that $f_{k}$ is a $k$-th key polynomial.

Proposition 5.1 Let $g \in \mathbf{K}[[x]][y]$ be a $k$-th key polynomial of $f$. Then $g$ is an irreducible (in $\mathbf{K}[[x]][y]$ ) distinguished polynomial. 


\section{Proof.}

Suppose that $g$ is not irreducible. Then $g=g_{1} g_{2}$ in $\mathbf{K}[[x]][y]$ with monic polynomials $g_{1}, g_{2}$ of positive degrees. Consequently, $\operatorname{deg}_{y} g_{1}, \operatorname{deg}_{y} g_{2}<$ $\operatorname{deg}_{y} g=\frac{n}{e_{k}}$ and by Property $\mathrm{I}_{k}$ we get $i_{0}\left(f, g_{1}\right), i_{0}\left(f, g_{2}\right) \in \mathbf{N} \overline{b_{0}}+\cdots+\mathbf{N} \overline{b_{k}}$ and $\overline{b_{k+1}}=i_{0}(f, g)=i_{0}\left(f, g_{1}\right)+i_{0}\left(f, g_{2}\right) \in \mathbf{N} \overline{b_{0}}+\cdots+\mathbf{N} \overline{b_{k}}$ which is a contradiction. Therefore $g$ is irreducible in $\mathbf{K}[[x]][y]$.

To check that $g=g(x, y) \in \mathbf{K}[[x]][y]$ is distinguished observe that from irreducibility of $g$ in $\mathbf{K}[[x]][y]$ and from Hensel's Lemma we get $g(0, y)=$ $(y-c)^{n / e_{k}}$ in $\mathbf{K}[y]$. On the other hand the condition $i_{0}(f, g)=\overline{b_{k+1}}$ implies $g(0,0)=0$ since $\overline{b_{k+1}}>0$. Hence $c=0$ and $g$ is a distinguished polynomial.

Corollary 5.2 The key polynomials of $f \in \mathbf{K}[[x, y]]$ are distinguished and irreducible in $\mathbf{K}[[x, y]]$.

Proof. The corollary follows from the fact that a distinguished polynomial irreducible in $\mathbf{K}[[x]][y]$ is irreducible in $\mathbf{K}[[x, y]]$ (see [A1], p. 75).

Lemma 5.3 Let $f=f(x, y) \in \mathbf{K}[[x, y]]$ be an irreducible power series such that $n=i_{0}(f, x)<+\infty$ and let $\left(\overline{b_{0}}, \ldots, \overline{b_{h}}\right)$ be an $n$-characteristic sequence. Suppose that there exist monic polynomials $f_{0}, \ldots, f_{h-1} \in \mathbf{K}[[x]][y]$ such that $\operatorname{deg}_{y} f_{k}=\frac{n}{e_{k}}$ and $i_{0}\left(f, f_{k}\right)=\overline{b_{k+1}}$ for $k \in\{0, \ldots, h-1\}$. Then $\overline{\operatorname{char}}_{x} f=$ $\left(\overline{b_{0}}, \ldots, \overline{b_{h}}\right)$ and $f_{0}, \ldots, f_{h-1}$ are key polynomials of $f$.

Proof. Recall that $\Gamma(f)=\left\{v_{f}(g): g \in \mathbf{K}[[x]][y] \backslash\{0\}: \operatorname{deg}_{y} g<n\right\}$. By Lemma 4.6 we get $\Gamma(f)=\mathbf{N} \overline{b_{0}}+\cdots+\mathbf{N} \overline{b_{h}}$. According to the first statement of Proposition 1.5 the sequence $\overline{b_{0}}, \ldots, \overline{b_{h}}$ is the $n$-minimal system of generators of the semigroup $\Gamma(f)$ and the lemma follows.

Proposition 5.4 Let $g$ be a $k$-th key polynomial of $f$. Then $g$ is a distinguished polynomial, irreducible in $\mathbf{K}[[x, y]]$ with characteristic $\overline{\operatorname{char}}_{x} g=$ $\left(\frac{\overline{b_{0}}}{e_{k}}, \ldots, \overline{\overline{b_{k}}}\right)$. Moreover the polynomials $f_{0}, f_{1}, \ldots, f_{k-1}$ are key polynomials of $g$.

Proof. We have already checked that the key polynomials are distinguished and irreducible. Let us calculate $i_{0}\left(f_{i}, g\right)$ for $i<k$. Consider $f_{i}, g, f$ and the log-distances $d_{x}\left(f_{i}, g\right)=\frac{e_{i} e_{k} i_{0}\left(f_{i}, g\right)}{n^{2}}, d_{x}\left(f_{i}, f\right)=\frac{e_{i} \overline{b_{i+1}}}{n^{2}}$ and $d_{x}(g, f)=\frac{e_{k} \overline{b_{k+1}}}{n^{2}}$. The sequence $\left(e_{i-1} \overline{b_{i}}\right)$ is strictly increasing, therefore $d_{x}\left(f_{i}, f\right)<d_{x}(g, f)$ and by the STI we get $d_{x}\left(f_{i}, g\right)=d_{x}\left(f_{i}, f\right)$ which implies $i_{0}\left(f_{i}, g\right)=\frac{\overline{b_{i+1}}}{e_{k}}$. 
On the other hand $\operatorname{deg}_{y} f_{i}=\frac{n}{e_{i}}=\frac{n}{e_{k}}: \frac{e_{i}}{e_{k}}$ and $\frac{e_{i}}{e_{k}}=\operatorname{gcd}\left(\frac{\overline{b_{0}}}{e_{k}}, \ldots, \frac{\overline{b_{i}}}{e_{k}}\right)$. The proposition follows from Lemma 5.3 .

We finish this section with

Proposition 5.5 Let $h \in \mathbf{K}[[x]][y]$ be a $(k-1)$-th key polynomial of $f$ and let $g \in \mathbf{K}[[x]][y]$ be a monic polynomial such that $\operatorname{deg}_{y} g=\frac{n}{e_{k}}$ and $v_{f}(g)>$ $n_{k} \overline{b_{k}}$. Let $g=h^{n_{k}}+a_{1} h^{n_{k}-1}+\cdots+a_{n_{k}}, \operatorname{deg}_{y} a_{i}<\operatorname{deg}_{y} h=\frac{n}{e_{k-1}}$ be the $h$-adic expansion of $g$. Then $v_{f}\left(a_{i}\right)>i \overline{b_{k}}$ if $1 \leq i<n_{k}$ and $v_{f}\left(a_{n_{k}}\right)=n_{k} \overline{b_{k}}$.

Proof. Consider the $h$-adic expansion of $g$

$$
g=h^{n_{k}}+a_{1} h^{n_{k}-1}+\cdots+a_{n_{k}},
$$

where $\operatorname{deg}_{y} a_{i}<\operatorname{deg}_{y} h=n / e_{k-1}$.

Let $I$ be the set of all $i \in\left\{1, \ldots, n_{k}\right\}$ such that $a_{i} \neq 0$. Since $v_{f}(g)>n_{k} \overline{b_{k}}=$ $v_{f}\left(h^{n_{k}}\right), I \neq \emptyset$. There is $v_{f}\left(a_{i}\right)<+\infty$ for $i \in I$ and by Property $\mathrm{I}_{k}$ we get $v_{f}\left(a_{i}\right) \in \mathbf{N} \overline{b_{0}}+\cdots+\mathbf{N} \overline{b_{k-1}}$, hence $v_{f}\left(a_{i}\right) \equiv 0 \bmod e_{k-1}$ for every $i \in I$. We have

$$
v_{f}\left(a_{i} h^{n_{k}-i}\right) \neq v_{f}\left(a_{j} h^{n_{k}-j}\right)
$$

for $i, j \in I$ with $i \neq j$.

Indeed, $v_{f}\left(a_{i} h^{n_{k}-i}\right)=v_{f}\left(a_{j} h^{n_{k}-j}\right)$ with $i<j$ implies, as in the proof of Property $\mathrm{I}_{k}$, the congruence $(j-i) \overline{b_{k}} / e_{k} \equiv 0 \bmod n_{k}$, which leads to a contradiction for $0<j-i<n_{k}$.

From (7) and (8) we have

$$
v_{f}\left(g-h^{n_{k}}\right)=\min _{i=1}^{n_{k}} v_{f}\left(a_{i} h^{n_{k}-i}\right) .
$$

By assumption $v_{f}(g)>n_{k} \overline{b_{k}}=v_{f}\left(h^{n_{k}}\right)$, so $v_{f}\left(g-h^{n_{k}}\right)=n_{k} \overline{b_{k}}$ and (9) implies $n_{k} \overline{b_{k}} \leq v_{f}\left(a_{i} h^{n_{k}-i}\right)=v_{f}\left(a_{i}\right)+\left(n_{k}-i\right) \overline{b_{k}}$ for $i \in\left\{1, \ldots, n_{k}\right\}$. Therefore we get

$$
v_{f}\left(a_{i}\right) \geq i \overline{b_{k}}
$$

for $i \in\left\{1, \ldots, n_{k}\right\}$.

Moreover,

$$
\text { if } v_{f}\left(a_{i}\right)=i \overline{b_{k}} \text { for } i \in\left\{1, \ldots, n_{k}\right\} \text { then } i=n_{k} \text {. }
$$

Indeed, from $v_{f}\left(a_{i}\right)=i \overline{b_{k}}$ it follows that $i \overline{b_{k}} \equiv 0 \bmod e_{k-1}$ and $i \overline{b_{k}} / e_{k} \equiv 0$ $\bmod n_{k}$, so $i \equiv 0 \bmod n_{k}$ because $\overline{b_{k}} / e_{k}$ and $n_{k}$ are coprime. Hence we 
get $i=n_{k}$. According to (9) there exists $i_{0} \in I$ such that $v_{f}\left(a_{i_{0}} h^{n_{k}-i_{0}}\right)=$ $v_{f}\left(g-h^{n_{k}}\right)=n_{k} \overline{b_{k}}$. Thus $v_{f}\left(a_{i_{0}}\right)=i_{0} \overline{b_{k}}$ and by (11) we get $i_{0}=n_{k}$.

\section{Notes}

Key polynomials of $f$ introduced in Mac, define curves of maximal contact with $\{f=0\}$ (see [LJ]) and are connected with curvettes associated with extremal points in the dual graph of $\{f=0\}$ (see, for example GB] p. 54, Po] p.13). They also play an important role in studying valuations $\mathrm{Sp}$.

\section{The Abhyankar-Moh theory}

We are going to prove the Abhyankar-Moh Theorem on approximate roots using the properties of key polynomials explained in Section 5. First let us recall the basic notions of Abhyankar-Moh theory (see [A-M1], [A3] or [Po]).

Let $R$ be an integral domain and let $d>1$ be a positive integer such that $d$ is a unit in $R$. Denote $\operatorname{deg} f:=\operatorname{deg}_{y} f$ the degree of the polynomial $f \in R[y]$ in one variable $y$ and assume that $d$ divides $\operatorname{deg} f$. According to Abhyankar and Moh ( $\mathrm{A}-\mathrm{M} 1$, Section 1) the approximate d-th root of $f$, denoted by $\sqrt[d]{f}$ is defined to be the unique monic polynomial satisfying $\operatorname{deg}\left(f-(\sqrt[d]{f})^{d}\right)<\operatorname{deg} f-\operatorname{deg} \sqrt[d]{f}$. For the existence and uniqueness of $\sqrt[d]{f}$ see [A-M1]. We put by convention $\sqrt[1]{f}=f$. Obviously $\operatorname{deg} \sqrt[d]{f}=\frac{\operatorname{deg} f}{d}$. From the definition it follows that $\sqrt[e]{\sqrt[d]{f}}=\sqrt[e d]{f}$ if $e d$ is a unit which divides $\operatorname{deg} f$ (see [Gw-Pt]).

Given any monic polynomial $g \in R[y]$ of $\operatorname{degree} \operatorname{deg} f / d$ we have the $g$-adic expansion of $f$, namely

$$
f=g^{d}+a_{1} g^{d-1}+\cdots+a_{d},
$$

where $a_{i} \in R[y], \operatorname{deg} a_{i}<\operatorname{deg} g$.

The polynomials $a_{i}$ are uniquely determined by $f$ and $g$.

The Tschirnhausen operator $\tau_{f}(g):=g+\frac{1}{d} a_{1}$ maps $g$ to $\tau_{f}(g)$ which is again a monic polynomial of degree $\operatorname{deg} f / d$. One checks (see [A-M1, Section 1 and Section 6) that

1. $a_{1}=0$ if and only if $g=\sqrt[d]{f}$,

2. if $f=\left(\tau_{f}(g)\right)^{d}+\overline{a_{1}}\left(\tau_{f}(g)\right)^{d-1}+\cdots+\overline{a_{d}}$ is the $\tau_{f}(g)$-expansion of $f$ then $\operatorname{deg} \overline{a_{1}}<\operatorname{deg} a_{1}$ or $\overline{a_{1}}=0$.

Using the above properties we get 
3. $\sqrt[d]{f}=\tau_{f}\left(\tau_{f} \cdots\left(\tau_{f}(g)\right)\right)$ with $\tau_{f}$ repeated $\operatorname{deg} f / d$ times.

Let $f=f(x, y) \in \mathbf{K}[[x]][y]$ be an irreducible distinguished polynomial of degree $n>1$ such that $\overline{\operatorname{char}}_{x} f=\left(\overline{b_{0}}, \ldots, \overline{b_{h}}\right), \overline{b_{0}}=n$.

Proposition 6.1 Let $g=g(x, y) \in \mathbf{K}[[x]][y]$ be a monic polynomial such that $\operatorname{deg}_{y} g=\frac{n}{e_{k}}$ and $v_{f}(g)>n_{k} \overline{b_{k}}$. Assume that $n_{k} \not \equiv 0 \bmod$ char $\mathbf{K}$. Then

(i) if $h$ is $a(k-1)$-th key polynomial of $f$ then $\tau_{g}(h)$ is a $(k-1)$-th key polynomial of $f$ as well,

(ii) $v_{f}\left(\sqrt[n_{k}]{g}\right)=\overline{b_{k}}$.

Proof. Consider the $h$-adic development of $g: g=h^{n_{k}}+a_{1} h^{n_{k}-1}+\cdots+$ $a_{n_{k}}$. By Proposition 5.5 we get $v_{f}\left(a_{1}\right)>\overline{b_{k}}$ (because $n_{k}>1$ ). Therefore $v_{f}\left(\tau_{g}(h)\right)=v_{f}\left(h+\frac{1}{n_{k}} a_{1}\right)=v_{f}(h)=\overline{b_{k}}$. Clearly $\operatorname{deg}_{y} \tau_{g}(h)=\operatorname{deg}_{y} h$ and $(i)$ follows.

To check (ii) use $\operatorname{deg}_{y} g / n_{k}=n / e_{k-1}$ times $(i)$ and the formula for the approximate root $\sqrt[n]{g} / g$ in terms of $\tau_{g}$.

Now we can prove the Abhyankar-Moh Theorem (see [A-M1]).

Theorem 6.2 (Abhyankar-Moh Fundamental Theorem on approximate roots)

Let $f=f(x, y) \in \mathbf{K}[[x]][y]$ be an irreducible distinguished polynomial of degree $n>1$ with $\overline{\operatorname{char}}_{x} f=\left(\overline{b_{0}}, \overline{b_{1}}, \ldots, \overline{b_{h}}\right)$ and $\overline{b_{0}}=v_{f}(x)=n$. Let $1 \leq k \leq h+1$. Suppose that $e_{k-1} \not \equiv 0 \bmod \operatorname{char} \mathbf{K}$. Then:

1. $v_{f}\left(\sqrt[e_{k-1}]{f}\right)=\overline{b_{k}}$,

2. $\sqrt[e_{k-1}]{f}$ is an irreducible distinguished polynomial of degree $n / e_{k-1}$ such that $\overline{\operatorname{char}}_{x} \sqrt[e_{k-1}]{f}=\left(\overline{b_{0}} / e_{k-1}, \overline{b_{1}} / e_{k-1}, \ldots, \overline{b_{k-1}} / e_{k-1}\right)$.

Proof. According to Proposition 5.4 it suffices to check the first part of the theorem. We use descendent induction on $k$. If $k=h+1$ then $e_{k-1}=e_{h}=1$, $\overline{b_{k}}=\overline{b_{h+1}}=+\infty$ and obviously $v_{f}(\sqrt[e h]{f})=\overline{b_{h+1}}$. Let $k \leq h$. Suppose that $e_{k} \neq \equiv 0(\bmod \operatorname{char} \mathbf{K})$ and $v_{f}\left(\sqrt[e_{k}]{f}\right)=\overline{b_{k+1}}$. The polynomial $\sqrt[e_{k}]{f}$ is of degree $n / e_{k}$ and $v_{f}\left(\sqrt[e_{k}]{f}\right)>n_{k} \overline{b_{k}}$ so we can apply Proposition 6.1 (ii) to $g=\sqrt[e_{k}]{f}$ to get $v_{f}\left(\sqrt[n_{k}]{g}\right)=\overline{b_{k}}$ provided that $n_{k} \not \equiv 0(\bmod$ char $\mathbf{K})$.

Assume that $e_{k-1} \not \equiv 0(\bmod \operatorname{char} \mathbf{K})$. Then $e_{k}, n_{k} \not \equiv 0(\bmod \operatorname{char} \mathbf{K})$ and we have $\sqrt[n_{k}]{g}=\sqrt[n_{k}]{\sqrt[e_{k}]{f}}=\sqrt[e_{k-1}]{f}$. Consequently, $v_{f}\left(\sqrt[e_{k-1}]{f}\right)=\overline{b_{k}}$ and we are done. 
Corollary 6.3 Suppose that $n \not \equiv 0(\bmod$ char $\mathbf{K})$. Then $\sqrt[e_{0}]{f}, \sqrt[e_{1}]{f}, \ldots$, $\sqrt[e h]{f}$ is a sequence of key polynomials of $f$.

We say that a projective plane curve $C$ defined over $\mathbf{K}$ has one branch at infinity if there is a line $L$ (line at infinity) intersecting $C$ in only one point $O$, and $C$ is analytically irreducible at $O$, that is the equation of $C$ in an affine coordinate system centered at $O$ is irreducible in the ring of formal power series.

In what follows we denote by $n$ the degree of $C$, by $n^{\prime}$ the multiplicity of $C$ at $O$ and we put $d:=\operatorname{gcd}\left(n, n^{\prime}\right)$.

We call $C$ permissible if $d \not \equiv 0(\bmod$ char $\mathbf{K})$.

Let us denote by $\Gamma_{O}$ the semigroup of the branch at infinity of the curve $C$. Since $C$ and $L$ intersect with multiplicity $n$ at $O$ there exists the $n$-minimal system of generators of $\Gamma_{O}$.

Theorem 6.4 (Abhyankar-Moh inequality) Assume that $C$ is a curve of degree $n>1$ with one branch at infinity and let $\left(\overline{b_{0}}, \ldots, \overline{b_{h}}\right)$ be the $n$ minimal system of generators of the semigroup $\Gamma_{O}$. If $C$ is permissible, then $e_{h-1} \overline{b_{h}}<n^{2}$.

Proof. Let $(x, y)$ be an affine coordinate system centered at $O$ such that $L$ has the equation $x=0$. Let $f(x, y)=0$ be a polynomial equation of $C$ of total degree $n$. Multiplying $f$ by a constant we may assume that $f$ is an $y$-distinguished polynomial (of degree $n$ since $C$ and $L$ intersect only at $O)$ irreducible in $\mathbf{K}[[x, y]]$. Let $\overline{\operatorname{char}_{x}} f=\left(\overline{b_{0}}, \ldots, \overline{b_{h}}\right)$. We have $\overline{b_{0}}=n$ and $\overline{b_{1}}=n^{\prime}$. Therefore $e_{1}=\operatorname{gcd}\left(\overline{b_{0}}, \overline{b_{1}}\right)=\operatorname{gcd}\left(n, n^{\prime}\right) \not \equiv 0(\bmod \operatorname{char} \mathbf{K})$ and consequently $e_{h-1} \not \equiv 0(\bmod \operatorname{char} \mathbf{K})$.

By Theorem 6.2 applied in the case $k=h$ the approximate root $\sqrt[e_{h}-1]{f}$ exists and $i_{0}\left(f, \sqrt[e_{h-1}]{f}\right)=\overline{b_{h}}$. One checks that the total degree of $\sqrt[e_{h-1}]{f}$ is $\frac{n}{e_{h-1}}$ (see, for example [Gw-P1], Remark on p. 201). Thus by Bézout's theorem applied to $f$ and $\sqrt[e_{h-1}]{f}$ we get $\overline{b_{h}}=i_{0}\left(f, \sqrt[e_{h-1}]{f}\right) \leq n \frac{n}{e_{h-1}}$. In fact, we have $\overline{b_{h}}<n \frac{n}{e_{h-1}}$ for $\overline{b_{h}}=n \frac{n}{e_{h-1}}$ would imply $\overline{b_{h}} \equiv 0\left(\bmod e_{h-1}\right)$ which is impossible.

The Abhyankar-Moh inequality implies an upper bound for the conductor $c$ of the semigroup $\Gamma_{O}$ :

Corollary 6.5 Under the notations and assumptions of Theorem 6.4.

$$
c \leq(n-1)^{2}-\left(n_{1}-1\right)\left(n-\overline{b_{1}}\right)=(n-1)^{2}-\left(\frac{n}{d}-1\right)\left(n-n^{\prime}\right) .
$$


Proof. By the Abhyankar-Moh inequality we have $e_{k-1} \overline{b_{k}}<n^{2}$ for $k \in$ $\{1, \ldots, h\}$ since the sequence $\left(e_{k-1} \overline{b_{k}}\right)$ is increasing. By the Conductor formula (Corollary 3.5) we get

$$
\begin{aligned}
c & =\sum_{k=1}^{h}\left(n_{k}-1\right) \overline{b_{k}}-\overline{b_{0}}+1=(n-1)^{2}-\sum_{k=1}^{h}\left(n_{k}-1\right)\left(\frac{n^{2}}{e_{k-1}}-\overline{b_{k}}\right) \\
& =(n-1)^{2}-\left(n_{1}-1\right)\left(n-\overline{b_{1}}\right)-\sum_{k=2}^{h}\left(n_{k}-1\right)\left(\frac{n^{2}}{e_{k-1}}-\overline{b_{k}}\right) \\
& \leq(n-1)^{2}-\left(n_{1}-1\right)\left(n-\overline{b_{1}}\right)=(n-1)^{2}-\left(\frac{n}{d}-1\right)\left(n-n^{\prime}\right) .
\end{aligned}
$$

The last equality follows from the fact that $\overline{b_{1}}=\min \left(\Gamma_{O} \backslash\{0\}\right)=$ mult $_{O} C=$ $n^{\prime}$.

Now we can prove

Theorem 6.6 (Abhyankar-Moh Embedding Line Theorem, first formulation)

Assume that $C$ is a rational projective irreducible curve of degree $n>1$ with one branch at infinity and such that the center of the branch at infinity $O$ is the unique singular point of $C$. Suppose that $C$ is permissible and let $n^{\prime}$ be the multiplicity of $C$ at $O$. Then $n-n^{\prime}$ divides $n$.

Proof. Let $c$ be the conductor of the semigroup $\Gamma_{O}$. Then we have

$$
c \leq(n-1)^{2}-\left(\frac{n}{d}-1\right)\left(n-n^{\prime}\right)
$$

by Corollary 6.5 .

On the other hand from the Noether formula for the genus of projective plane curve we get

$$
c=(n-1)(n-2) .
$$

Combining (12) and (13) we obtain $\left(\frac{n}{d}-1\right)\left(n-n^{\prime}\right) \leq n-1<n$ and $\left(\frac{n}{d}-1\right)\left(\frac{n}{d}-\frac{n^{\prime}}{d}\right)<\frac{n}{d}$. Therefore we get $\frac{n}{d}-\frac{n^{\prime}}{d}=1$ and $n-n^{\prime}$ divides $n$.

Remark 6.7 From the proof of Theorem 6.6 we also conclude that $\operatorname{gcd}\left(n, n^{\prime}\right)=$ $n-n^{\prime}$. 
Remark 6.8 Let us keep the assumptions of Theorem 6.6 and let $\overline{\beta_{0}}, \overline{\beta_{1}}, \ldots$ be the minimal sequence of generators of the semigroup $\Gamma_{O}$. Then $n^{\prime}=\overline{\beta_{0}}$ and since $n$ is the intersection multiplicity of the branch at infinity with a nonsingular branch we have $n \leq \overline{\beta_{1}}$ and $n \equiv 0\left(\bmod \overline{\beta_{0}}\right)$ if $n \neq \overline{\beta_{1}}$. We claim that if $n \neq \overline{\beta_{1}}$ then $n=2 \overline{\beta_{0}}$. Indeed, if $n \neq \overline{\beta_{1}}$ then $n=a \overline{\beta_{0}}$ for an integer $a>0$ and $n=b\left(n-\overline{\beta_{0}}\right)$ for an integer $b>0$ by Theorem [6.6. Thus we get $a=(a-1) b$ which implies $a=2$.

We say that a nonsingular projective curve $C^{\prime}$ has maximal contact with $C$ at infinity if $C^{\prime}$ intersects $C$ at 0 with multiplicity $\overline{\beta_{1}}$ (see [LJ]]). The line at infinity has maximal contact with $C$ if and only if $n \neq 2 n^{\prime}$. If $n=2 n^{\prime}$ then there exists a nonsingular curve $C^{\prime}$ of degree 2 which has maximal contact with $C$ at infinity (if $f=0$ is the affine equation of $C$ such that in the proof of Theorem 6.4 then $C^{\prime}$ is given by the equation $\sqrt[n^{\prime}]{f}=0$ ).

A polynomial mapping $F=(P, Q): \mathbf{K} \longrightarrow \mathbf{K}^{2}$ is called a polynomial embedding (of the line $\mathbf{K}$ ) if there is a polynomial $G: \mathbf{K}^{2} \longrightarrow \mathbf{K}$ such that $G \circ F$ is equal to the identity of $\mathbf{K}$.

Theorem 6.9 (Abhyankar-Moh Embedding Line Theorem, second formulation) If $F=(P, Q): \mathbf{K} \longrightarrow \mathbf{K}^{2}$ is a polynomial embedding such that $m=\operatorname{deg} P$, $n=\operatorname{deg} Q>0$ and $\operatorname{gcd}(m, n) \not \equiv 0(\bmod \operatorname{char} \mathbf{K})$ then $m$ divides $n$ or $n$ divides $m$.

Proof. We may assume that $1<m<n$. Let $C$ be the projective closure of the affine curve $F\left(\mathbf{K}^{2}\right)$. Then $C$ is irreducible, rational with one branch at infinity centered at $O=(0: 1: 0)$. Moreover $C$ is of degree $n=\operatorname{deg} Q$ and its multiplicity at $O$ is $n^{\prime}=\operatorname{deg} Q-\operatorname{deg} P=n-m$. Therefore $C$ is permissible. Apply Theorem 6.6 to the curve $C$.

\section{Notes}

S.S. Abhyankar and T.T. Moh developed the theory of approximate roots of polynomials with coefficients in the meromorphic series field $\mathbf{K}((x))$ in the fundamental paper [A-M1]. In [A-M2 they applied approximate roots to prove the Embedding Line Theorem. Later on Abhyankar in [A3] gave a simplified version of A-M1 and A-M2. The approach of Abhyankar and Moh is based on the technique of deformations of power series. H. Pinkham in $\mathrm{Pi}$ proposed a method of eliminating the deformations which works in the algebroid case $\mathbf{K}[[x]][y]$. P. Russel in $[\mathbf{R u}]$ used the Hamburger-Noether expansions to reprove the Abhyankar-Moh results (in the algebroid case) with weaker assumptions on the field characteristic. In our presentation of 
the subject we followed Gw-Pt] (see also Chan-W], Chan]). The reader will find in $[\mathrm{Po}]$ more references on the approximate roots. The assumption $C$ is permissible in the Embedding Line Theorem is relevant (see [N]).

\section{A formula for the intersection multiplicity of two branches}

The aim of this section is to give a new formula for the intersection multiplicity of two branches.

Let $\{f=0\}$ and $\{g=0\}$ be two branches different from $\{x=0\}$. Let $\overline{\operatorname{char}_{x}} f=\left(\overline{b_{0}}, \ldots, \overline{b_{h}}\right), \overline{b_{0}}=n=i_{0}(f, x)$ and $\overline{\operatorname{char}_{x}} g=\left(\overline{b_{0}^{\prime}}, \ldots, \overline{b_{h^{\prime}}^{\prime}}\right), \overline{b_{0}^{\prime}}=$ $n^{\prime}=i_{0}(g, x)$. We denote by $f_{0}, \ldots, f_{h}$ and $g_{0}, \ldots, g_{h^{\prime}}$ key polynomials of $f$ and $g$, respectively.

Lemma 7.1 The equalities $\frac{\overline{b_{i}}}{n}=\frac{\overline{b_{i}^{\prime}}}{n^{\prime}}$ for all $i \in\{1, \ldots, k\}$ imply $\frac{n}{e_{i}}=\frac{n^{\prime}}{e_{i}^{\prime}}$ and $\frac{\overline{b_{i}}}{e_{i}}=\frac{\overline{b_{i}^{\prime}}}{e_{i}^{\prime}}$ for all $i \in\{1, \ldots, k\}$.

Proof. We get $n e_{i}^{\prime}=n \operatorname{gcd}\left(\overline{b_{0}^{\prime}}, \ldots, \overline{b_{i}^{\prime}}\right)=\operatorname{gcd}\left(n \overline{b_{0}^{\prime}}, \ldots, n \overline{b_{i}^{\prime}}\right)=n^{\prime} e_{i}$. Thus $\frac{n}{e_{i}}=\frac{n^{\prime}}{e_{i}^{\prime}}$ and consequently $\frac{\overline{b_{i}}}{e_{i}}=\frac{\overline{b_{i}^{\prime}}}{e_{i}^{\prime}}$ for all $i \in\{1, \ldots, k\}$ since $\frac{\overline{b_{i}}}{n}=\frac{\overline{b_{i}^{\prime}}}{n^{\prime}}$.

Theorem 7.2 Let $n=i_{0}(f, x)>1$ and suppose that $\frac{i_{0}(f, g)}{i_{0}(x, g)}>\frac{e_{k-1} \overline{b_{k}}}{n}$ for an integer $k \in\{1, \ldots, h\}$. Then $k \leq h^{\prime}$ and $\frac{\overline{b_{i}}}{n}=\frac{\overline{b_{i}^{\prime}}}{n^{\prime}}$ for all $i \in\{1, \ldots, k\}$. The first $k$ key polynomials $f_{0}, \ldots, f_{k-1}$ of $f$ are the first $k$ key polynomials of $g$.

Proof.

Let us start with

Property $7.3 n i_{0}\left(g, f_{i-1}\right)=n^{\prime} \overline{b_{i}}$ for $i \in\{1, \ldots, k\}$.

Proof. Fix $i \in\{1, \ldots, k\}$ and consider the power series $f, f_{i-1}$ and $g$. We have $d_{x}\left(f, f_{i-1}\right)=\frac{e_{i-1} \overline{b_{i}}}{n^{2}}, d_{x}(f, g)=\frac{i_{0}(f, g)}{n n^{\prime}}>\frac{e_{k-1} \overline{b_{k}}}{n^{2}}$ (by assumption) and $d_{x}\left(g, f_{i-1}\right)=\frac{e_{i-1} i_{0}\left(g, f_{i-1}\right)}{n n^{\prime}}$. Since $d_{x}\left(f, f_{i-1}\right)<d_{x}(f, g)$ by the STI we get $d_{x}\left(g, f_{i-1}\right)=d_{x}\left(f, f_{i-1}\right)$, which implies the property.

Property $7.4 n^{\prime} \equiv 0\left(\bmod \frac{n}{e_{k}}\right)$. 
Proof. We may write $e_{k}=a_{0} \overline{b_{0}}+a_{1} \overline{b_{1}}+\cdots+a_{k} \overline{b_{k}}$ with $a_{0}, \ldots, a_{k} \in \mathbf{Z}$ since $e_{k}=\operatorname{gcd}\left(\overline{b_{0}}, \ldots, \overline{b_{k}}\right)$. Hence we get $e_{k} n^{\prime}=\left(a_{0} n^{\prime}\right) n+a_{1}\left(n^{\prime} \overline{b_{1}}\right)+\cdots+a_{k}\left(n^{\prime} \overline{b_{k}}\right) \equiv$ $0(\bmod n)$ by Property 7.3 and consequently $n^{\prime} \equiv 0\left(\bmod \frac{n}{e_{k}}\right)$.

Property 7.5 Let $i>0$ be an integer. Then $d_{x}\left(g, f_{i-1}\right)=\frac{e_{i-1} \overline{b_{i}}}{n^{2}}$ for $i \leq k$, $d_{x}\left(g, g_{i-1}\right)=\frac{e_{i-1}^{\prime} \overline{b_{i}^{\prime}}}{\left(n^{\prime}\right)^{2}}$ for $i \leq h^{\prime}$, and $d_{x}\left(f_{i-1}, g_{i-1}\right)=\frac{e_{i-1} e_{i-1}^{\prime} i_{0}\left(f_{i-1}, g_{i-1}\right)}{n n^{\prime}}$ for $i \leq \min \left(k, h^{\prime}\right)$.

Proof. We have $d_{x}\left(g, f_{i-1}\right)=\frac{e_{i-1} i_{0}\left(g, f_{i-1}\right)}{n^{\prime} n}=\frac{e_{i-1} \overline{b_{i}}}{n^{2}}$ by Property 7.3. The formulae for $d_{x}\left(g, g_{i-1}\right)$ and $d_{x}\left(f_{i-1}, g_{i-1}\right)$ follow from the definitions.

Property 7.6 We have $h^{\prime} \geq 1$ and $\overline{\frac{b_{1}}{n}}=\frac{\overline{b_{1}^{\prime}}}{n^{\prime}}$.

Proof. From Property 7.4 it follows $n^{\prime}>1$ since $\frac{n}{e_{k}}>1$ for $k>0$. Thus $h^{\prime} \geq 1$ and we may apply Property 7.5 for $i=1$. We get $d_{x}\left(g, f_{0}\right)=\frac{\overline{b_{1}}}{n} \notin \mathbf{N}$, $d_{x}\left(g, g_{0}\right)=\frac{\overline{b_{1}^{\prime}}}{n^{\prime}} \notin \mathbf{N}$ and $d_{x}\left(f_{0}, g_{0}\right)=i_{0}\left(f_{0}, g_{0}\right) \in \mathbf{N}$. By the STI we obtain $\overline{\frac{b_{1}}{n}}=\overline{\overline{b_{1}^{\prime}}}$.

Property 7.7 Let $i>0$ be an integer such that $i<k, i \leq h^{\prime}$ and $\frac{\overline{b_{j}}}{n}=\frac{\overline{b_{j}^{\prime}}}{n^{\prime}}$ for all $j \leq i$. Then $i<h^{\prime}$ and $\overline{\frac{b_{i+1}}{n}}=\frac{\overline{b_{i+1}^{\prime}}}{n^{\prime}}$.

Proof. From the assumption $\frac{\overline{b_{j}}}{n}=\frac{\overline{b_{j}^{\prime}}}{n^{\prime}}$ for all $j \leq i$ and from Lemma 7.1 we get $\frac{e_{i}}{n}=\frac{e_{i}^{\prime}}{n^{\prime}}$. By Property 7.4 we may write $n^{\prime}=l \frac{n}{e_{k}}$, where $l>0$ is an integer. Thus $e_{i}^{\prime}=n^{\prime} \frac{e_{i}}{n}=l \frac{e_{i}}{e_{k}}>1$ since $i<k$. From $e_{i}^{\prime}>1$ we get obviously $i<h^{\prime}$. Now we may apply Property 7.5 for the index $i+1$ since $i+1 \leq k$ and $i+1 \leq h^{\prime}$. We get $d_{x}\left(g, f_{i}\right)=\frac{e_{i} \overline{b_{i+1}}}{n^{2}}, d_{x}\left(g, g_{i}\right)=$ $\frac{e_{i}^{\prime} \overline{b_{i+1}^{\prime}}}{\left(n^{\prime}\right)^{2}}$ and $d_{x}\left(f_{i}, g_{i}\right)=\left(\frac{e_{i}}{n}\right)\left(\frac{e_{i}^{\prime}}{n^{\prime}}\right) i_{0}\left(f_{i}, g_{i}\right)$. Recall that $\frac{e_{i}}{n}=\frac{e_{i}^{\prime}}{n^{\prime}}$. Note that $d_{x}\left(g, f_{i}\right) \neq d_{x}\left(f_{i}, g_{i}\right)$. Indeed if we had $d_{x}\left(g, f_{i}\right)=d_{x}\left(f, g_{i}\right)$ then we would get $\overline{b_{i+1}}=e_{i} i_{0}\left(f_{i}, g_{i}\right)$ which is impossible since $\overline{b_{i+1}} \not \equiv 0\left(\bmod e_{i}\right)$. Similarly we check that $d_{x}\left(g, g_{i}\right) \neq d_{x}\left(f_{i}, g_{i}\right)$. Using the STI we get $d_{x}\left(g, f_{i}\right)=d_{x}\left(g, g_{i}\right)$, which implies $\frac{\overline{b_{i+1}}}{n}=\frac{\overline{b_{i+1}^{\prime}}}{n^{\prime}}$.

Now we can finish the proof of Theorem 7.2

From Properties 7.6 and 7.7 we conclude that $k \leq h^{\prime}$ and $\frac{\overline{b_{i}}}{n}=\frac{\overline{b_{i}^{\prime}}}{n^{\prime}}$ for $i \in$ $\{1, \ldots, k\}$, which proves the first part of Theorem 7.2 , Let $i \in\{0,1, \ldots, k-$ 
1\}. By Property $7.3 i_{0}\left(g, f_{i-1}\right)=\frac{n^{\prime} \overline{b_{i}}}{n}=\overline{b_{i}^{\prime}}$ since $\frac{\overline{b_{i}}}{n}=\frac{\overline{b_{i}^{\prime}}}{n^{\prime}}$. Moreover $\operatorname{deg}_{y}\left(f_{i-1}\right)=\frac{n}{e_{i-1}}=\frac{n^{\prime}}{e_{i-1}^{\prime}}$ so $f_{i-1}$ is a key-polynomial of $g$.

Remark 7.8 Under the notations and assumptions of Theorem 7.2, we get

$$
\frac{i_{0}(g, f)}{i_{0}(x, f)}=\frac{i_{0}(f, g)}{i_{0}(x, g)} \frac{i_{0}(x, g)}{i_{0}(x, f)}>\frac{e_{k-1} \overline{b_{k}}}{n} \frac{n^{\prime}}{n}=\frac{e_{k-1}^{\prime}}{n^{\prime}} \frac{\overline{b_{k}^{\prime}}}{n^{\prime}} n^{\prime}=\frac{e_{k-1}^{\prime} \overline{b_{k}^{\prime}}}{n^{\prime}} .
$$

Let $f, g \in \mathbf{K}[[x, y]]$ be irreducible power series such that the branches $\{f=$ $0\}$ and $\{g=0\}$ are different from $\{x=0\}$. We put, by definition:

$$
k_{x}(f, g)=\min \left\{k>0: \frac{i_{0}(f, g)}{i_{0}(x, g)} \leq \frac{e_{k-1} \overline{b_{k}}}{n}\right\} .
$$

Obviously, we have $1 \leq k_{x}(f, g) \leq h+1$. Recall that $\overline{b_{h+1}}=+\infty$ so $k_{x}(f, g)=h+1$ if and only if $\frac{i_{0}(f, g)}{i_{0}(x, g)}>\frac{e_{h-1} \overline{b_{h}}}{n}$.

On the other hand $k_{x}(f, g)=1$ means that $\frac{i_{0}(f, g)}{i_{0}(x, g)} \leq \overline{b_{1}}$.

Note that $k_{x}(f, g)$ is different from the coincidence exponent defined by means of Puiseux' expansions in the case of characteristic zero (see $[\mathrm{Po}$, p. 299, Gw-P1, p. 205).

Lemma 7.9 Suppose that $\frac{\overline{b_{1}}}{n} \neq \frac{\overline{b_{1}^{\prime}}}{n^{\prime}}$. Then $i_{0}(f, g) \leq \inf \left\{n^{\prime} \overline{b_{1}}, n \overline{b_{1}^{\prime}}\right\}$. If $i_{0}(f, g)<\inf \left\{n^{\prime} \overline{b_{1}}, n \overline{b_{1}^{\prime}}\right\}$ then $i_{0}(f, g)=n n^{\prime} i_{0}\left(f_{0}, g_{0}\right)$.

Proof. If $\frac{\overline{b_{1}}}{n} \neq \overline{\frac{b_{1}^{\prime}}{n^{\prime}}}$ then $\overline{b_{1}} \neq+\infty$ or $\overline{b_{1}^{\prime}} \neq+\infty$ that is $n>1$ or $n^{\prime}>1$. We may assume $n>1$. If $f$ and $g$ have a common key-polynomial of degree 1 then $i_{0}(f, g)=\inf \left\{n^{\prime} \overline{b_{1}}, n \overline{b_{1}^{\prime}}\right\}$. Indeed, if $f_{0}$ is a key-polynomial of $f$ and $g$ then $d_{x}\left(f, f_{0}\right)=\frac{\overline{b_{1}}}{n}$ and $d_{x}\left(g, f_{0}\right)=\frac{\overline{b_{1}^{\prime}}}{n^{\prime}}$. From the assumption $\frac{\overline{b_{1}}}{n} \neq \frac{\overline{b_{1}^{\prime}}}{n^{\prime}}$ we get $d_{x}(f, g)=\inf \left\{\frac{\overline{b_{1}}}{n}, \overline{\frac{b_{1}^{\prime}}{n^{\prime}}}\right\}$ by the STI whence $i_{0}(f, g)=\inf \left\{n \overline{b_{1}}, n^{\prime} \overline{b_{1}^{\prime}}\right\}$.

If $f$ and $g$ do not have a common key-polynomial of degree 1 then $i_{0}\left(f, g_{0}\right)<$ $\overline{b_{1}}$ and $i_{0}\left(g, f_{0}\right)<\overline{b_{1}^{\prime}}$. Consequently we get $d_{x}\left(f, g_{0}\right)<\frac{\overline{b_{1}}}{n}, d_{x}\left(f, f_{0}\right)=\frac{\overline{b_{1}}}{n}$ and $d_{x}\left(f_{0}, g_{0}\right)=d_{x}\left(f, g_{0}\right)<\frac{\overline{b_{1}}}{n}$ by the STI. Analogously applying the STI to $g, f_{0}$ and $g_{0}$ we get $d_{x}\left(f_{0}, g_{0}\right)=d_{x}\left(f_{0}, g\right)<\frac{\overline{b_{1}^{\prime}}}{n^{\prime}}$. We may assume without loss of 
generality that $\inf \left\{n \overline{b_{1}^{\prime}}, n^{\prime} \overline{b_{1}}\right\}=n \overline{b_{1}^{\prime}}$. Then $\frac{\overline{b_{1}^{\prime}}}{n^{\prime}}<\frac{\overline{b_{1}}}{n}$ and $d_{x}\left(g, f_{0}\right)<d_{x}\left(f, f_{0}\right)$. By the STI we get $d_{x}(f, g)=d_{x}\left(g, f_{0}\right)$. Thus $d_{x}(f, g)=d_{x}\left(g, f_{0}\right)<\frac{\overline{b_{1}^{\prime}}}{n^{\prime}}$ and $i_{0}(f, g)<n n^{\prime} \frac{\overline{b_{1}^{\prime}}}{n^{\prime}}=n \overline{b_{1}^{\prime}}$. On the other hand $d_{x}(f, g)=d_{x}\left(g, f_{0}\right)=d_{x}\left(f_{0}, g_{0}\right)$, which gives $i_{0}(f, g)=n n^{\prime} i_{0}\left(f_{0}, g_{0}\right)$.

Lemma 7.10 If $k_{x}(f, g)=k$ then $k \leq h^{\prime}+1$ and $\frac{i_{0}(f, g)}{i_{0}(f, x)} \leq \frac{e_{k-1}^{\prime} \overline{b_{k}^{\prime}}}{n^{\prime}}$.

Proof. Let $k=1$. Then $\frac{i_{0}(f, g)}{i_{0}(g, x)} \leq \overline{b_{1}}$. If $\frac{\overline{b_{1}}}{n}=\frac{\overline{b_{1}^{\prime}}}{n^{\prime}}$ then

$$
\frac{i_{0}(f, g)}{i_{0}(f, x)}=\frac{i_{0}(g, x)}{i_{0}(f, x)} \frac{i_{0}(f, g)}{i_{0}(g, x)} \leq\left(\frac{n^{\prime}}{n}\right) \overline{b_{1}}=n^{\prime}\left(\overline{\frac{b_{1}^{\prime}}{n^{\prime}}}\right)=\overline{b_{1}^{\prime}} .
$$

If $\frac{\overline{b_{1}}}{n} \neq \overline{\overline{b_{1}^{\prime}}}$ then $i_{0}(f, g) \leq \inf \left\{n^{\prime} \overline{n_{1}}, n \overline{b_{1}^{\prime}}\right\} \leq n \overline{b_{1}^{\prime}}$ by Lemma 7.9 and consequently $\frac{i_{0}(f, g)}{i_{0}(x, f)} \leq \overline{b_{1}^{\prime}}$.

Now suppose that $k>1$. By definition of $k_{x}(f, g)$ of $f$ and $g$ we get

$$
\frac{i_{0}(f, g)}{i_{0}(x, g)}>\frac{e_{k-2} \overline{b_{k-1}}}{n} \text {. }
$$

By Theorem 7.2 we get $\frac{\overline{b_{i}}}{n}=\frac{\overline{b_{i}^{\prime}}}{n^{\prime}}$ for $i \leq k-1 \leq h^{\prime}$. Moreover $f_{0}, \ldots, f_{k-2}$ are key polynomials of $g$. If $e_{k-1}^{\prime}=1$ then $h^{\prime}=k-1, \overline{b_{k}^{\prime}}=\overline{b_{h^{\prime}+1}^{\prime}}=+\infty$ and the lemma is obvious. Suppose that $e_{k-1}^{\prime}>1$. Then $\overline{b_{k}^{\prime}}<+\infty$. If we suppose that Lemma 7.10 is not true then

$$
\frac{i_{0}(f, g)}{i_{0}(x, f)}>\frac{e_{k-1}^{\prime} \overline{b_{k}^{\prime}}}{n^{\prime}}
$$

Applying Theorem 7.2 to $g$ and $f$ we get that $k-1<h^{\prime}$, that is $k \leq h^{\prime}$ and $\frac{\overline{b_{i}}}{n}=\frac{\overline{b_{i}^{\prime}}}{n^{\prime}}$ for $i \leq k$. So in particular $\frac{\overline{b_{k}}}{n}=\frac{\overline{b_{k}^{\prime}}}{n^{\prime}}$ and by the definition of $k$ we get $\frac{i_{0}(f, g)}{i_{0}(g, x)} \leq \frac{e_{k-1} \overline{b_{k}}}{n}=e_{k-1}\left(\frac{\overline{b_{k}^{\prime}}}{n^{\prime}}\right)$ and $\frac{i_{0}(f, g)}{n} \leq \frac{n^{\prime}}{n} e_{k-1} \frac{\overline{b_{k}^{\prime}}}{n^{\prime}}=n^{\prime} \frac{e_{k-1}^{\prime}}{n^{\prime}} \frac{\overline{b_{k}^{\prime}}}{n^{\prime}}=\frac{e_{k-1}^{\prime} \overline{b_{k}^{\prime}}}{n^{\prime}}$ which is a contradiction with inequality (15).

Theorem 7.11 (Formula for the intersection multiplicity) Let $f, g \in$ $\mathbf{K}[[x, y]]$ be irreducible power series such that $n=i_{0}(f, x)<+\infty$ and $n^{\prime}=$ $i_{0}(g, x)<+\infty$. Let $\overline{\operatorname{char}_{x}} f=\left(\overline{b_{0}}, \ldots, \overline{b_{h}}\right), \overline{b_{0}}=n$ and $\overline{\operatorname{char}_{x}} g=\left(\overline{b_{0}^{\prime}}, \ldots, \overline{b_{h^{\prime}}^{\prime}}\right)$, $\overline{b_{0}^{\prime}}=n^{\prime}$ be the Seidenberg characteristic sequences of the branches $\{f=0\}$ and $\{g=0\}$. Let $k=k_{x}(f, g)$. Then we have 
1. $\frac{\overline{b_{i}}}{n}=\frac{\overline{b_{i}^{\prime}}}{n^{\prime}}$, for $i<k$,

2. $i_{0}(f, g) \leq \inf \left\{e_{k-1}^{\prime} \overline{b_{k}}, e_{k-1} \overline{b_{k}^{\prime}}\right\}$.

Let $\left(f_{0}, \ldots, f_{h}\right)$ (resp. $\left.\left(g_{0}, \ldots, g_{h^{\prime}}\right)\right)$ be a sequence of key polynomials of $f$ (resp. of $g)$. We get

3. If $i_{0}(f, g)<\inf \left\{e_{k-1}^{\prime} \overline{b_{k}}, e_{k-1} \overline{b_{k}^{\prime}}\right\}$ then $i_{0}(f, g)=e_{k-1} e_{k-1}^{\prime} i_{0}\left(f_{k-1}, g_{k-1}\right)$,

4. if $k>1$ then $e_{k-2}^{\prime} \overline{b_{k-1}}=e_{k-2} \overline{b_{k-1}^{\prime}}$ and $i_{0}(f, g)>e_{k-2}^{\prime} \overline{b_{k-1}}$.

Moreover $f_{0}, \ldots, f_{k-1}$ are the first $k$ key polynomials of $g$ and $g_{0}, \ldots, g_{k-1}$ are the first $k$ key polynomials of $f$.

Proof. Part (1) of the theorem follows immediately from Theorem 7.2 , From (1) we conclude, by Lemma 7.1, that $\frac{n}{e_{k-1}}=\frac{n^{\prime}}{e_{k-1}^{\prime}}$.

By the definition of $k_{x}(f, g)$ we get

$$
i_{0}(f, g) \leq i_{0}(x, g) \frac{e_{k-1} \overline{b_{k}}}{n}=n^{\prime} \frac{e_{k-1} \overline{b_{k}}}{n}=e_{k-1}^{\prime} \overline{b_{k}}
$$

On the other hand, by Lemma 7.1 we get

$$
i_{0}(f, g) \leq i_{0}(x, f) \frac{e_{k-1}^{\prime} \overline{b_{k}^{\prime}}}{n^{\prime}}=n \frac{e_{k-1}^{\prime} \overline{b_{k}^{\prime}}}{n^{\prime}}=e_{k-1} \overline{b_{k}^{\prime}}
$$

Combining the above inequalities we obtain Part (2) of the theorem.

To check Part (3) suppose that $i_{0}(f, g)<\inf \left\{e_{k-1}^{\prime} \overline{b_{k}}, e_{k-1} \overline{b_{k}^{\prime}}\right\}$. Then we have $\frac{i_{0}(f, g)}{i_{0}(x, g)}<\frac{e_{k-1} \overline{b_{k}}}{n}$ and $\frac{i_{0}(f, g)}{i_{0}(x, f)}<\frac{e_{k-1}^{\prime} \overline{b_{k}^{\prime}}}{n^{\prime}} d_{x}(f, g)<\frac{e_{k-1} \overline{b_{k}}}{n^{2}}=d_{x}\left(f, f_{k-1}\right)$ and $d_{x}(f, g)<\frac{e_{k-1}^{\prime} \overline{b_{k}^{\prime}}}{\left(n^{\prime}\right)^{2}}=d_{x}\left(g, g_{k-1}\right)$. By the STI applied to $f, f_{k-1}, g$ and to $f, g_{k-1}, g$ we get $d_{x}(f, g)=d_{x}\left(f, g_{k-1}\right)=d_{x}\left(g, f_{k-1}\right)<d_{x}\left(f, f_{k-1}\right), d_{x}\left(g, g_{k-1}\right)$. From $d_{x}(f, g)=d_{x}\left(g, f_{k-1}\right)<d_{x}\left(g, g_{k-1}\right)$ we conclude, by the STI applied to $f_{k-1}, g, g_{k-1}$ that $d_{x}(f, g)=d_{x}\left(f_{k-1}, g_{k-1}\right)$, which implies $i_{0}(f, g)=$ $e_{k-1} e_{k-1}^{\prime} i_{0}\left(f_{k-1}, g_{k-1}\right)$. This proves Part (3).

To check Part (4) suppose that $k>1$. Note that $\frac{e_{k-2}^{\prime} \overline{b_{k-1}}}{n^{\prime} n}=\left(\frac{e_{k-2}^{\prime}}{n^{\prime}}\right)\left(\frac{\overline{b_{k-1}}}{n}\right)=$ $\left(\frac{e_{k-2}}{n}\right)\left(\overline{\frac{b_{k-1}^{\prime}}{n^{\prime}}}\right)$ whence $e_{k-2}^{\prime} \overline{b_{k}}=e_{k-2} \overline{b_{k}^{\prime}}$. Since $k>1$ we get, by definition 
of $k_{x}(f, g)$, that $\frac{i_{0}(f, g)}{i_{0}(x, g)}>\frac{e_{k-2} \overline{b_{k-1}}}{n}$, which implies by the first part of the theorem the inequality $i_{0}(f, g)>e_{k-2}^{\prime} \overline{b_{k-1}}$.

The assertion on the key polynomials follows from the second part of Theorem 7.2 .

Theorem 7.12 Let $\{f=0\}$ and $\{g=0\}$ be two different branches and let $\{l=0\}$ be a smooth branch. Suppose that $n=i_{0}(f, l)<+\infty, n^{\prime}=i_{0}(g, l)<$ $+\infty$ and let $d=\operatorname{gcd}\left(n, n^{\prime}\right)$. Then $i_{0}(f, g) \equiv 0 \bmod \left(\frac{n}{d}\right.$ or $\left.\frac{n^{\prime}}{d}\right)$.

Proof. We may assume that $n, n^{\prime}>1$ and $l=x$. Let $k=k_{x}(f, g)$. By Theorem 7.2 and Lemma 7.1 we get

$$
\frac{n}{e_{i}}=\frac{n^{\prime}}{e_{i}^{\prime}} \text { for } i<k
$$

On the other hand from the second and third part of Theorem 7.11 it follows

$$
i_{0}(f, g) \equiv 0 \bmod \left(e_{k-1} \text { or } e_{k-1}^{\prime}\right) .
$$

From (16) we get

$$
e_{k-1} \equiv 0 \quad\left(\bmod \frac{n}{d}\right) \text { and } e_{k-1}^{\prime} \equiv 0 \quad\left(\bmod \frac{n^{\prime}}{d}\right) .
$$

Now (17) and (18) imply the theorem.

Using Theorem 7.12 we will prove the following basic property of polynomial automorphisms of the plane (see [J] and $\mathrm{vdK}]$ ).

Theorem 7.13 (Jung-van der Kulk) Let the mapping $(P, Q): \mathbf{K}^{2} \longrightarrow$ $\mathbf{K}^{2}$ be a polynomial automorphism. Then of the two integers $m=\operatorname{deg} P$, $n=\operatorname{deg} Q$ one divides the other.

Proof. (see $\mathrm{vdK}$ ) Let $C$ and $D$ be projective curves with affine equations $P=0$ and $Q=0$. Then $\operatorname{deg} D=n, \operatorname{deg} C=m$ and each of the curves $C, D$ has exactly one branch at infinity. By Bézout's Theorem these branches intersect with multiplicity $i=m n-1$. The line at infinity cuts the branches of $C$ and $D$ with multiplicities $m$ and $n$ respectively. Thus by Theorem 7.12 we get $i \equiv 0 \quad\left(\bmod \frac{m}{d}\right.$ or $\left.\frac{n}{d}\right)$, where $d=\operatorname{gcd}(m, n)$. This implies that $m$ divides $n$ or $n$ divides $m$, since $i=m n-1$.

\section{Notes}


The classical formula for the intersection multiplicity of two branches (see $[\mathrm{He}$, Chapter 8 or $[\mathrm{Po}$ ) was well-known to geometers of the nineteenth century: H.J.S. Smith, G.H. Halphen and M. Noether. It was used by Zariski in Za2 to study the saturation of the local rings. Ancochea gave in Anc a formula for the intersection multiplicity of two branches in terms of Hamburger-Noether expansions (see also [Ru], Cam1, [Del]).

\section{The Abhyankar-Moh irreducibility criterion}

Let $f \in \mathbf{K}[[x, y]]$ be an irreducible power series such that $\{f=0\} \neq\{x=0\}$. Let $n=i_{0}(f, x)>1$ and $\overline{\operatorname{char}_{x}} f=\left(\overline{b_{0}}, \ldots, \overline{b_{h}}\right), \overline{b_{0}}=n$.

Lemma 8.1 Let $g=g(x, y) \in \mathbf{K}[[x, y]]$ be an irreducible power series such that $\{g=0\} \neq\{x=0\}$ and let $k$ be an integer such that $1 \leq k \leq h$. If $\frac{i_{0}(f, g)}{i_{0}(g, x)}>\frac{e_{k-1} \overline{b_{k}}}{n}$ then $i_{0}(g, x) \equiv 0\left(\bmod \frac{n}{e_{k}}\right)$. If, additionally, $i_{0}(g, x)=\frac{n}{e_{k}}$ then $\overline{\operatorname{char}_{x}} g=\left(\frac{\overline{b_{0}}}{e_{k}}, \ldots, \frac{\overline{b_{k}}}{e_{k}}\right)$.

Proof. It follows immediately from Theorem 7.2 and Lemma 7.1

Theorem 8.2 Let $g \in \mathbf{K}[[x, y]]$ be a power series such that $i_{0}(g, x)=\frac{n}{e_{k}}$ and $i_{0}(f, g)>n_{k} \overline{b_{k}}$ for $a k \in\{1, \ldots, h\}$. Then $g$ is irreducible and $\overline{\text { char }_{x}} g=$ $\left(\frac{\overline{b_{0}}}{e_{k}}, \ldots, \frac{\overline{b_{k}}}{e_{k}}\right)$.

Proof. Suppose that $i_{0}(f, g)>n_{k} \overline{b_{k}}$ and let $g=g_{1} \cdots g_{s}$ with irreducible $g_{j} \in \mathbf{K}[[x, y]]$, for $j \in\{1, \ldots, s\}$. Then there exists $j \in\{1, \ldots, s\}$ such that

$$
\frac{i_{0}\left(f, g_{j}\right)}{i_{0}\left(g_{j}, x\right)}>\frac{e_{k-1} \overline{b_{k}}}{n}
$$

Indeed, suppose that inequality (19) is not true. Then $i_{0}\left(f, g_{j}\right) \leq \frac{e_{k-1} \overline{b_{k}}}{n} i_{0}\left(g_{j}, x\right)$ for all $j \in\{1, \ldots, s\}$ and we get $i_{0}(f, g)=\sum_{j=1}^{s} i_{0}\left(f, g_{j}\right) \leq \sum_{j=1}^{s} \frac{e_{k-1} \overline{b_{k}}}{n} i_{0}\left(g_{j}, x\right)=$ $\frac{e_{k-1} \overline{b_{k}}}{n} i_{0}(g, x)=n_{k} \overline{b_{k}}$ which contradicts the assumption about $i_{0}(f, g)$. The inequality (19) implies by Lemma 8.1 that $i_{0}\left(g_{j}, x\right)=q \frac{n}{e_{k}}$ for some integer $q>0$. On the other hand $i_{0}\left(g_{j}, x\right) \leq i_{0}(g, x)=\frac{n}{e_{k}}$. Therefore $q=1$ and $i_{0}\left(g_{j}, x\right)=i_{0}(g, x)$. Recall that $g_{j}$ divides $g, g_{j}$ is irreducible and ord $g_{j}(0, y)=$ ord $g(0, y)$, thus $g_{j}$ is associated with $g$, which proves the irreducibility of $g$. We get $\overline{\operatorname{char}_{x}} g=\left(\frac{\overline{b_{0}}}{e_{k}}, \ldots, \frac{\overline{b_{k}}}{e_{k}}\right)$ from the second part of Lemma 8.1 . 
Corollary 8.3 (Abhyankar-Moh irreducibility criterion) If $i_{0}(g, x)=$ $n$ and $i_{0}(f, g)>n_{h} \overline{b_{h}}$ then $g$ is irreducible and $\overline{\operatorname{char}_{x}} g=\overline{\operatorname{char}_{x}} f$.

Using the Abhyankar-Moh inequality and the irreducibility criterion we prove

Theorem 8.4 (Moh-Ephraim Pencil Theorem) Assume that $C$ is a plane curve of degree $n>1$ with one branch at infinity and that $C$ is permissible. Let $D$ be another plane curve of degree $n>1$ with one point at infinity $O$ which is the unique common point of $C$ and $D$. Then

1. the curve $D$ has one branch at infinity,

2. the branches at infinity of the curves $C$ and $D$ are equisingular.

Proof. Let $(x, y)$ be an affine coordinate system centered at $O$ such that the line at infinity has the equation $x=0$. Let $f(x, y)=0$ and $g(x, y)=0$ be the polynomial equations of the curves $C$ and $D$. Then $i_{0}(f, x)=i_{0}(g, x)=n$ and $i_{0}(f, g)=n^{2}$ by Bézout's theorem. Let $\overline{\operatorname{char}_{x}} f=\left(\overline{b_{0}}, \ldots, \overline{b_{h}}\right)$. By the Abhyankar-Moh inequality we have $e_{h-1} \overline{b_{h}}<i_{0}(f, g)$. Now, the assertions follow from Theorem 8.2 (case $k=h$ ).

Remark 8.5 Suppose that the plane curve $C$ of the Moh-Ephraim pencil theorem is given by the homogeneous equation $F(X, Y, Z)=0$ of degree $n$ and let $L: Z=0$ be the line at infinity. Consider the pencil $C_{\lambda}: F(X, Y, Z)-$ $\lambda Z^{n}=0, \lambda \in \mathbf{K}$. Applying Theorem 8.4 to $C$ and $D=C_{\lambda}, \lambda \neq 0$ we prove that the pencil $C_{\lambda}$ is equisingular at infinity.

\section{Notes}

The Abhyankar-Moh irreducibility criterion was proved in [A-M1] (Lemma 3.4) and explained in details in A3] (Theorem 12.4). The original version of the criterion was given for meromorphic curves. Using Puiseux series the authors had to assume $n \not \equiv 0(\bmod \operatorname{char} \mathbf{K})$. The version of the criterion presented in this paper is borrowed from [Gw-Pl] where the result is proved for the case char $\mathbf{K}=0$.

The first part (irreducibility) of the Moh-Ephraim Pencil Theorem is due to Moh [Moh2, the second part (equisingularity) to Ephraim [E] (see also Chan]). In our treatment of the subject we do not need the assumption char $\mathbf{K}=0$, which is necessary in the quoted papers. 


\section{Characterization of the semigroups associated with branches}

In this section we give a new proof of the well-known theorem on the existence of branches with given semigroup (see Bre and Ang]). Following Teissier [Te1] we give explicitly the equation of a plane curve with given characteristic. Our proof is written in the spirit of this paper, we do not use the technique of deformations. Here is the main result of this section.

Theorem 9.1 Let $\left(\overline{b_{0}}, \ldots, \overline{b_{h}}\right)$ be an $n$-characteristic sequence. Suppose there exists a distinguished irreducible polynomial $f_{h-1} \in \mathbf{K}[[x]][y]$ such that $\overline{\operatorname{char}_{x}} f_{h-1}=\left(\frac{\overline{b_{0}}}{e_{h-1}}, \ldots, \frac{\overline{b_{h-1}}}{e_{h-1}}\right)$. Let $f_{0}, \ldots, f_{h-2} \in \mathbf{K}[[x]][y]$ be a sequence of key polynomials of $f_{h-1}$. Let $a_{0}, \ldots, a_{h-1}$ be the (unique) sequence of integers such that $a_{0} \overline{b_{0}}+a_{1} \overline{b_{1}}+\cdots+a_{h-1} \overline{b_{h-1}}=n_{h} \overline{b_{h}}$, where $0<a_{0}$ and $0 \leq a_{i}<n_{i}$ for $i \in\{1, \ldots, h-1\}$ and let $c \in \mathbf{K} \backslash\{0\}$. Put $f_{h}=f_{h-1}^{n_{h}}+c x^{a_{0}} f_{0}^{a_{1}} \cdots f_{h-2}^{a_{h-1}}$. Then

1. $f_{h}$ is a distinguished irreducible polynomial of degree $n$, that is, $i_{0}\left(f_{h}, x\right)=$ $\operatorname{deg}_{y} f_{h}$,

2. $\overline{\operatorname{char}_{x}} f_{h}=\left(\overline{b_{0}}, \ldots, \overline{b_{h}}\right)$ and $f_{0}, \ldots, f_{h-1}$ are key polynomials of $f_{h}$.

Proof. Since $f_{h-1}$ is a distinguished polynomial of degree $\frac{n}{e_{h-1}}$ and $a_{0}>0$, we have

$$
\begin{aligned}
i_{0}\left(f_{h}, x\right) & =i_{0}\left(f_{h-1}^{n_{h}}+c x^{a_{0}} f_{0}^{a_{1}} \cdots f_{h-2}^{a_{h-1}}, x\right)=i_{0}\left(f_{h-1}^{n_{h}}, x\right) \\
& =n_{h} i_{0}\left(f_{h-1}, x\right)=n_{h} \frac{n}{e_{h-1}}=n .
\end{aligned}
$$

To calculate $\operatorname{deg}_{y} f_{h}$ observe that $\operatorname{deg}_{y} f_{h-1}^{n_{h}}=n_{h} \operatorname{deg}_{y} f_{h-1}=n_{h} \frac{n}{e_{h-1}}=n$ and $\operatorname{deg}_{y} c x^{a_{0}} f_{0}^{a_{1}} \cdots f_{h-2}^{a_{h-1}}=a_{1} \frac{n}{e_{0}}+\cdots+a_{h-1} \frac{n}{e_{h-2}} \leq\left(n_{1}-1\right) \frac{n}{e_{0}}+\cdots+$ $\left(n_{h-1}-1\right) \frac{n}{e_{h-2}}=\frac{n}{e_{h-1}}-1<n$. Therefore we get $\operatorname{deg}_{y} f_{h}=n$. The proof that $f_{h}$ is irreducible is harder. We need auxiliary lemmas.

Lemma $9.2 i_{0}\left(f_{h}, f_{h-1}\right)=\overline{b_{h}}$.

Proof. 


$$
\begin{aligned}
i_{0}\left(f_{h}, f_{h-1}\right) & =i_{0}\left(f_{h-1}^{n_{h}}+c x^{a_{0}} f_{0}^{a_{1}} \cdots f_{h-2}^{a_{h-1}}, f_{h-1}\right)=i_{0}\left(x^{a_{0}} f_{0}^{a_{1}} \cdots f_{h-2}^{a_{h-1}}, f_{h-1}\right) \\
& =a_{0} i_{0}\left(x, f_{h-1}\right)+a_{1} i_{0}\left(f_{0}, f_{h-1}\right)+\cdots+a_{k-1} i_{0}\left(f_{h-2}, f_{h-1}\right) \\
& =a_{0} \frac{\overline{b_{0}}}{e_{h-1}}+a_{1} \frac{\overline{b_{1}}}{e_{h-1}}+\cdots+a_{h-1} \frac{\overline{b_{h-1}}}{e_{h-1}}=\frac{1}{e_{h-1}} n_{h} \overline{b_{h}}=\overline{b_{h}} .
\end{aligned}
$$

Lemma 9.3 There exists an irreducible factor $\phi$ of $f_{h}$ such that

$$
\frac{i_{0}\left(f_{h-1}, \phi\right)}{i_{0}(\phi, x)}>\frac{n_{h-1} \overline{b_{h-1}}}{n} .
$$

Proof. Let $f_{h}=\phi_{1} \cdots \phi_{s}$ with irreducible factors $\phi_{i} \in \mathbf{K}[[x, y]]$ for $i \in$ $\{1, \ldots, s\}$. Suppose that $\frac{i_{0}\left(f_{h-1}, \phi\right)}{i_{0}(\phi, x)} \leq \frac{n_{h-1} \overline{b_{h-1}}}{n}$ for all $i \in\{1, \ldots, s\}$. By Lemma 9.2 we get

$$
\begin{aligned}
\overline{b_{h}} & =i_{0}\left(f_{h}, f_{h-1}\right)=\sum_{i=1}^{s} i_{0}\left(\phi_{i}, f_{h-1}\right) \leq \sum_{i=1}^{s} \frac{n_{h-1} \overline{b_{h-1}}}{n} i_{0}\left(\phi_{i}, x\right) \\
& =\frac{n_{h-1} \overline{b_{h-1}}}{n} \sum_{i=1}^{s} i_{0}\left(\phi_{i}, x\right)=\frac{n_{h-1} \overline{b_{h-1}}}{n} i_{0}\left(f_{h}, x\right)=n_{h-1} \overline{b_{h-1}}<\overline{b_{h}}
\end{aligned}
$$

which is a contradiction.

Lemma 9.4 Let $\phi$ be an irreducible factor of $f_{h}$ such that $\frac{i_{0}\left(f_{h-1}, \phi\right)}{i_{0}(\phi, x)}>$ $\frac{n_{h-1} \overline{b_{h-1}}}{n}$. Then there exists $\nu \in\left\{1, \ldots, n_{h}\right\}$ such that $i_{0}(\phi, x)=\nu \frac{\overline{b_{0}}}{e_{h-1}}$ and $i_{0}\left(\phi, f_{k}\right)=\nu \frac{\overline{b_{k+1}}}{e_{h-1}}$ for $k<h-1$.

Proof. Recall that $\overline{\operatorname{char}_{x}} f_{h-1}=\left(\frac{\overline{b_{0}}}{e_{h-1}}, \ldots, \frac{\overline{b_{h-1}}}{e_{h-1}}\right)$. Applying Lemma 8.1 to the irreducible power series $f_{h-1}$ and $\phi$ (note that $\frac{n_{h-1} \overline{b_{h-1}}}{n}=\frac{n_{h-1} \frac{\overline{b_{h-1}}}{e_{h-1}}}{\frac{n}{e_{h-1}}}$ ) we conclude that $i_{0}(\phi, x) \equiv 0\left(\bmod \frac{n}{e_{h-1}}\right)$. Therefore we can write $i_{0}(\phi, x)=$ $\nu \frac{n}{e_{h-1}}$ with $\nu \leq e_{h-1}=n_{h}$ since $i_{0}(\phi, x) \leq i_{0}\left(f_{h}, x\right)=n$. Fix $k<h-1$ and consider the three branches $f_{k}=0, f_{h-1}=0$ and $\phi=0$. We get $d_{x}\left(f_{k}, \phi\right)=$ $\frac{e_{h-1} e_{k} i_{0}\left(f_{k}, \phi\right)}{\nu n^{2}}, d_{x}\left(f_{h-1}, \phi\right)=\frac{i_{0}\left(f_{h-1}, \phi\right)}{i_{0}(\phi, x) \frac{n}{e_{h-1}}}>\frac{n_{h-1} \overline{b_{h-1}}}{n} \frac{e_{h-1}}{n}=\frac{e_{h-2} \overline{b_{h-1}}}{n^{2}}$, and $d_{x}\left(f_{h-1}, f_{k}\right)=\frac{\overline{b_{k+1}} / e_{h-1}}{\left(n / e_{h-1}\right)\left(n / e_{k}\right)}=\frac{e_{k} \overline{b_{k+1}}}{n^{2}} \leq \frac{e_{h-2} \overline{b_{h-1}}}{n^{2}}$, for $k<h-1$. Therefore 
$d_{x}\left(f_{h-1}, f_{k}\right)<d_{x}\left(f_{h-1}, \phi\right)$ and by the STI we get $d_{x}\left(f_{h-1}, f_{k}\right)=d_{x}\left(f_{k}, \phi\right)$, which implies $i_{0}\left(f_{k}, \phi\right)=\nu \frac{\overline{b_{k+1}}}{e_{h-1}}$.

Now we are in a position to check that $f_{h}$ is an irreducible power series. Let $\phi$ be an irreducible factor of $f_{h}$ such that in Lemma 9.3. Since $f_{h}=f_{h-1}^{n_{h}}+$ $c x^{a_{0}} f_{0}^{a_{1}} \cdots f_{h-2}^{a_{h-1}}$ and $\phi$ is an irreducible factor of $f_{h}$ we get $i_{0}\left(f_{h-1}^{n_{h}}, \phi\right)=$ $i_{0}\left(x^{a_{0}} f_{0}^{a_{1}} \cdots f_{h-2}^{a_{h-1}}, \phi\right)$. Therefore, by Lemma 9.4 we have

$$
\begin{aligned}
n_{h} i_{0}\left(f_{h-1}, \phi\right) & =a_{0} i_{0}(x, \phi)+a_{1} i_{0}\left(f_{0}, \phi\right)+\cdots+a_{h-1} i_{0}\left(f_{h-2}, \phi\right) \\
& =a_{0} \nu \frac{\overline{b_{0}}}{e_{h-1}}+a_{1} \nu \frac{\overline{b_{1}}}{e_{h-1}}+\cdots+a_{h-1} \nu \frac{\overline{b_{h-1}}}{e_{h-1}}=\frac{\nu}{e_{h-1}} n_{h} \overline{b_{h}}=\nu \overline{b_{h}} .
\end{aligned}
$$

Since $\nu \overline{b_{h}} \equiv 0\left(\bmod n_{h}\right)$ and $\overline{b_{h}}, n_{h}=e_{h-1}$ are coprime we get $\nu \equiv 0(\bmod$ $\left.n_{h}\right)$ and $\nu=n_{h}$ because $1 \leq \nu \leq n_{h}$.

From Lemma 9.4 we get $i_{0}(\phi, x)=n_{h} \frac{\overline{b_{0}}}{e_{h-1}}=\overline{b_{0}}=n=i_{0}\left(f_{h}, x\right)$. Since $\phi$ divides $f_{h}$ we get $f_{h}=\phi \psi$ in $\mathbf{K}[[x, y]]$ with $\psi(0) \neq 0$. Therefore $f_{h}$ is irreducible.

Now we prove the second statement of the theorem. First we check that $i_{0}\left(f_{h}, f_{k}\right)=\overline{b_{k+1}}$ for $k \in\{0,1, \ldots, h-1\}$. We have $i_{0}\left(f_{h}, f_{h-1}\right)=\overline{b_{h}}$ by Lemma 9.2. Therefore we may assume that $h>1$ and $k<h-1$. Applying Lemma 9.4 to the power series $\phi=f_{h}$ we get $i_{0}\left(f_{h}, f_{k}\right)=n_{h} \frac{\overline{b_{k+1}}}{e_{h-1}}=\overline{b_{k+1}}$ since $\nu=n_{h}$. Recall that $\operatorname{deg}_{y} f_{k}=\frac{n}{e_{k}}$ for $k \in\{0,1, \ldots, h-1\}$. Using Lemma 5.3 we conclude that $\overline{\operatorname{char}_{x}} f_{h}=\left(\overline{b_{0}}, \ldots, \overline{b_{h}}\right)$ and that $f_{0}, \ldots, f_{h-1}$ is a sequence of key polynomials of $f_{h}$.

Theorem 9.5 (Bresinsky-Angermüller) Let $\overline{b_{0}}, \ldots, \overline{b_{h}}$ be a sequence of positive integers. Then the following two conditions are equivalent:

1. There is an irreducible power series $f \in \mathbf{K}[[x]][y]$ such that $i_{0}(f, x)=$ $\overline{b_{0}}$ and $\overline{b_{0}}, \ldots, \overline{b_{h}}$ is the $\overline{b_{0}}$-minimal sequence of generators of the semigroup $\Gamma(f)$.

2. The numbers $\overline{b_{0}}, \ldots, \overline{b_{h}}$ form a $\overline{b_{0}}$-characteristic sequence.

Proof. The implication (1) $\Longrightarrow(2)$ follows from the Semigroup Theorem (Theorem 3.2). To check that $(2) \Longrightarrow(1)$ we proceed by induction on the length $h$ of the characteristic sequence using Theorem 9.1. If $h=0$ then $\left(\overline{b_{0}}\right)=(1)$ and we take $f=y$. Let $h>0$ and suppose that the implication $(2) \Longrightarrow(1)$ is true for $h-1$. Then there exists an irreducible distinguished 
polynomial $f_{h-1} \in \mathbf{K}[[x]][y]$ such that $\Gamma\left(f_{h-1}\right)=\frac{\overline{b_{0}}}{e_{h-1}} \mathbf{N}+\cdots+\frac{\overline{b_{h-1}}}{e_{h-1}} \mathbf{N}$. Let $f_{0}, \ldots, f_{h-2}$ be a sequence of key polynomials of $f_{h-1}$. Take $f=f_{h-1}^{n_{h}}+$ $x^{a_{0}} f_{0}^{a_{1}} \cdots f_{h-2}^{a_{h-1}}$, where $0<a_{0}$ and $0 \leq a_{i}<n_{i}$ for $i \in\{1, \ldots, h\}$ is the (unique) sequence of integers such that $a_{0} \overline{b_{0}}+a_{1} \overline{b_{1}}+\cdots+a_{h-1} \overline{b_{h-1}}=n_{h} \overline{b_{h}}$. Then by Theorem $9.1 f$ is an irreducible power series and $\Gamma(f)=\overline{b_{0}} \mathbf{N}+$ $\cdots+\overline{b_{h}} \mathbf{N}$.

Let $\left(\overline{b_{0}}, \ldots, \overline{b_{h}}\right)$ be an $n$-characteristic sequence. For any $k \in\{1, \ldots, h\}$ we have Bézout's relation $n_{k} \overline{b_{k}}=a_{k 0} \overline{b_{0}}+a_{k 1} \overline{b_{1}}+\cdots+a_{k k-1} \overline{b_{k-1}}$, where $a_{k 0}>0$ and $0 \leq a_{k i}<n_{i}$ for $i \in\{1, \ldots, k-1\}$. Take $c_{1}, \ldots, c_{h} \in \mathbf{K} \backslash\{0\}$ and define in a recurrent way the polynomials $g_{0}, \ldots, g_{h}$ by putting $g_{0}=y$, $g_{1}=g_{0}^{n_{1}}+c_{1} x^{a_{10}}=y^{n / e_{1}}+c_{1} x^{\overline{b_{1}} / e_{1}}, \ldots, g_{h}=g_{h-1}^{n_{h}}+c_{h} x^{a_{h 0}} g_{0}^{a_{h 1}} \cdots g_{h-2}^{a_{h h-1}}$.

Theorem 9.6 (cf. [Te1] and [Re]) The polynomials $g_{0}, \ldots, g_{h}$ are distinguished and irreducible. We have $\overline{\text { char }_{x}} g_{k}=\left(\frac{\overline{b_{0}}}{e_{k}}, \ldots, \frac{\overline{b_{h}}}{e_{k}}\right)$. The sequence $g_{0}, \ldots g_{k-1}$ is a sequence of key polynomials of $g_{k}$.

Proof. The theorem follows from Theorem 9.1 by induction on $k$.

\section{Notes}

Theorem 9.5 characterizing the semigroups associated with branches is due to Bresinsky Bre (the case of characteristic 0) and to Angermüller Ang (the case of arbitrary characteristic, see also Gar-St]). Both authors consider only generic case, i.e. $i_{0}(f, x)=$ ord $f$. Theorem 9.6 which gives an explicit equation of the branch with given semigroup was obtained by Teissier by the method of deformations of the monomial curve associated with a branch. Another proof was given by Reguera López in [Re].

\section{Description of branches with given semigroup}

We need two preliminary lemmas.

Lemma 10.1 Let $f \in \mathbf{K}[[x]][y]$ be a distinguished irreducible polynomial of degree $n>0$. Suppose that $\overline{\operatorname{char}_{x}} f=\left(\overline{b_{0}}, \ldots, \overline{b_{h}}\right), \overline{b_{0}}=n$ and let $f_{0}, f_{1}, \ldots, f_{h-1}$ be a sequence of key polynomials of $f$. Then any polynomial $g \in \mathbf{K}[[x]][y]$ of $y$-degree strictly less than $n$ has a unique expansion of the form

$$
g=\sum g_{\alpha_{1}, \ldots, \alpha_{h}} f_{0}^{\alpha_{1}} \cdots f_{h-1}^{\alpha_{h}}, \quad g_{\alpha_{1}, \ldots, \alpha_{h}} \in \mathbf{K}[[x]],
$$

where $0 \leq \alpha_{1}<n_{1}, \ldots, 0 \leq \alpha_{h}<n_{h}$. Moreover 
1. the $y$-degrees of the terms appearing in the right-hand side of the preceding equality are all distinct,

2. $i_{0}(f, g)=\inf \left\{\left(\operatorname{ord} g_{\alpha_{1}, \ldots, \alpha_{h}}\right) n+\alpha_{1} \overline{b_{1}}+\cdots+\alpha_{h} \overline{b_{h}}: 0 \leq \alpha_{i}<n_{i}\right.$ for $i=$ $1, \ldots, h\}$.

Proof. The existence and unicity of the expansion and the inequality for the degrees holds for polynomials with coefficients in arbitrary integral domain (see A3, Section 2). The formula for the intersection multiplicity follows from the observation that the intersection multiplicities $i_{0}\left(f, g_{\alpha_{1}, \ldots, \alpha_{h}} f_{0}^{\alpha_{1}} \cdots f_{h-1}^{\alpha_{h}}\right)=\left(\operatorname{ord} g_{\alpha_{1}, \ldots, \alpha_{h}}\right) n+\alpha_{1} \overline{b_{1}}+\cdots+\alpha_{h} \overline{b_{h}}$ are pairwise distinct by the unicity of Bézout's relation.

Lemma 10.2 Under the notation and assumptions introduced above, if $\operatorname{deg}_{y} g<$ $n / e_{k}$ then $i_{0}(f, g)=e_{k} i_{0}\left(f_{k}, g\right)$.

Proof. Suppose that $\operatorname{deg}_{y} g<n / e_{k}$. Then by Lemma 10.1 we get

$$
g=\sum g_{\alpha_{1}, \ldots, \alpha_{h}} f_{0}^{\alpha_{1}} \cdots f_{h-1}^{\alpha_{h}},
$$

where $0 \leq \alpha_{i}<n_{i}$, for $i \in\{1, \ldots, h\}$. Since $\operatorname{deg}_{y} g<n / e_{k}$ we have, by the first statement of Lemma 10.1, $\alpha_{k+1}=\cdots=\alpha_{h}=0$.

By Proposition 5.4 $f_{k}$ is an irreducible distinguished polynomial, $\overline{\operatorname{char}_{x}} f_{k}=$ $\left(\frac{\overline{b_{0}}}{e_{k}}, \ldots, \overline{b_{k}}\right)$ and $f_{0}, \ldots, f_{k-1}$ are key polynomials of $f_{k}$. Therefore there exist $\alpha_{1}, \ldots, \alpha_{k}$ such that

$$
i_{0}\left(f_{k}, g\right)=\left(\operatorname{ord} g_{\alpha_{1}, \ldots, \alpha_{k}, 0, \ldots, 0}\right) \frac{n}{e_{k}}+\alpha_{1} \frac{\overline{b_{1}}}{e_{k}}+\cdots+\alpha_{k} \frac{\overline{b_{k}}}{e_{k}}=\frac{1}{e_{k}} i_{0}(f, g)
$$

and the lemma follows.

Let $\phi, f \in \mathbf{K}[[x]][y]$ be distinguished polynomials such that $N=\frac{\operatorname{deg}_{y} f}{\operatorname{deg}_{y} \phi}$ is a positive integer. Consider the $\phi$-adic expansion of $f$ :

$$
f=\phi^{N}+\alpha_{1} \phi^{N-1}+\cdots+\alpha_{N}, \operatorname{deg}_{y} \alpha_{i}<\operatorname{deg}_{y} \phi \text { for } i \in\{1, \ldots, N\} .
$$

Put $\alpha_{0}=1$ and $I=\left\{i \in[0, N]: i_{0}\left(\alpha_{i}, \phi\right) \neq+\infty\right\}$. We define the Newton polygon $\Delta_{x, \phi}(f)$ of $f$ with respect to the pair $(x, \phi)$ by setting

$$
\Delta_{x, \phi}(f)=\text { convex } \bigcup_{i \in I}\left\{\left(i_{0}\left(\alpha_{i}, \phi\right), N-i\right)+\mathbf{R}_{\geq 0}^{2}\right\} .
$$


The polygon $\Delta_{x, \phi}(f)$ intersects the vertical axis in the point $(0, N)$ and the horizontal axis in the point $\left(i_{0}(f, \phi), 0\right)$ provided that $i_{0}(f, \phi) \neq+\infty$. If $\phi=y$ then $\Delta_{x, \phi}(f)=\Delta_{x, y}(f)$ is the usual Newton polygon of $f$ in coordinates $(x, y)$.

In the sequel we use Teissier's notation (see [Te3]): for any integers $k, l>0$ we put

$$
\left\{\begin{array}{l}
\frac{k}{\bar{l}} \\
\}
\end{array}=\operatorname{convex}\left\{\left((k, 0)+\mathbf{R}_{\geq 0}^{2}\right) \cup\left((0, l)+\mathbf{R}_{\geq 0}^{2}\right)\right\}\right.
$$

Proposition 10.3 If $f \in \mathbf{K}[[x, y]]$ is an irreducible power series, $\overline{\text { char }_{x}} f=$ $\left(\overline{b_{0}}, \ldots, \overline{b_{h}}\right)$ and $f_{0}, f_{1}, \ldots, f_{h}$ is a sequence of key polynomials of $f$ then

$$
\Delta_{x, f_{k-1}}\left(f_{k}\right)=\left\{\frac{\overline{b_{k}} / e_{k}}{\overline{\overline{e_{k-1}} / e_{k}}}\right\} .
$$

Proof. The $f_{k-1}$-adic expansion of $f_{k}$ is of the form $f_{k}=f_{k-1}^{n_{k}}+a_{1} f_{k-1}^{n_{k-1}}+$ $\cdots+a_{n_{k}}$, where $\operatorname{deg}_{y} a_{i}<\frac{n}{e_{k-1}}$ for $i \in\left\{1, \ldots, n_{k}\right\}$. By Proposition 5.5 we have $i_{0}\left(f, a_{i}\right)>i \overline{b_{k}}$ for $0<i<n_{k}$ and $i_{0}\left(f, a_{n_{k}}\right)=n_{k} \overline{b_{k}}$. By Lemma 10.2 we get $i_{0}\left(f, a_{i}\right)=e_{k-1} i_{0}\left(f_{k-1}, a_{i}\right)$ for $0<i \leq n_{k}$. Therefore we have $i_{0}\left(f_{k-1}, a_{i}\right)>i \frac{\overline{b_{k}}}{e_{k-1}}$ for $0<i<n_{k}$ and $i_{0}\left(f_{k-1}, a_{n_{k}}\right)=\frac{\overline{b_{k}}}{e_{k}}$, which implies $\Delta_{x, f_{k-1}}\left(f_{k}\right)=\left\{\frac{\overline{b_{k}} / e_{k}}{\overline{e_{k-1} / e_{k}}}\right\}$.

Proposition 10.4 Let $f$ be an irreducible distinguished polynomial of degree $n>1$. Let $\overline{\operatorname{char}_{x}} f=\left(\overline{b_{0}}, \ldots, \overline{b_{h}}\right)$ and let $\phi \in \mathbf{K}[[x]][y]$ be a $(h-1)$-key polynomial of $f$. Then

1. $\overline{\operatorname{char}_{x}} \phi=\left(\frac{\overline{b_{0}}}{e_{h-1}}, \ldots, \frac{\overline{b_{h-1}}}{e_{h-1}}\right)$,

2. $\Delta_{x, \phi}(f)=\left\{\frac{\overline{b_{h}}}{\overline{e_{h-1}}}\right\}$.

Proof. The proposition follows from Propositions 5.4 and 10.3 ,

The following theorem is the main result of this section.

Theorem 10.5 Let $\left(\overline{b_{0}}, \ldots, \overline{b_{h}}\right)$ be an $n$-characteristic sequence $(n>1)$. Let $f \in \mathbf{K}[[x]][y]$ be a distinguished polynomial of degree $n$ for which there exists an irreducible distinguished polynomial $\phi \in \mathbf{K}[[x]][y]$ such that 


$$
\begin{aligned}
& \text { 1. } \overline{\operatorname{char}_{x}} \phi=\left(\frac{\overline{b_{0}}}{e_{h-1}}, \ldots, \overline{\frac{b_{h-1}}{e_{h-1}}}\right), \\
& \text { 2. } \Delta_{x, \phi}(f)=\left\{\frac{\overline{b_{h}}}{\overline{e_{h-1}}}\right\} .
\end{aligned}
$$

Then $f$ is irreducible, $\overline{\operatorname{char}_{x}} f=\left(\overline{b_{0}}, \ldots, \overline{b_{h}}\right)$ and $\phi$ is a key polynomial of $f$.

Proof. Let $\phi_{0}, \ldots, \phi_{h-2}, \phi_{h-1}=\phi$ be a sequence of key polynomials of $\phi$.

From the assumption about $\Delta_{x, \phi}(f)$ it follows that if $f=\phi^{n_{h}}+\alpha_{1} \phi^{n_{h}-1}+$ $\cdots+\alpha_{n_{h}}, \operatorname{deg}_{y} \alpha_{i}<\frac{n}{e_{h-1}}$ for $i \in\left\{1, \ldots, n_{h}\right\}$ is the $\phi$-adic expansion of $f$ then

$$
\text { (1) } i_{0}(f, \phi)=i_{0}\left(\alpha_{n_{h}}, \phi\right)=\overline{b_{h}},
$$

and

$$
\text { (2) } i_{0}\left(\alpha_{i}, \phi\right)>i \frac{\overline{b_{h}}}{n_{h}} \text { for } 0<i<n_{h}
$$

(note that $\operatorname{gcd}\left(n_{h}, \overline{b_{h}}\right)=\operatorname{gcd}\left(e_{h-1}, \overline{b_{h}}\right)=e_{h}=1$ whence the strict inequality in $(2))$.

There exists a unique sentence of integers $l_{0}, \ldots, l_{h-1}$ such that $l_{0} \overline{b_{0}}+\cdots+$ $l_{h-1} \overline{b_{h-1}}=e_{h-1} \overline{b_{h}}$, where $l_{0}>0$ and $0 \leq l_{i}<n_{i}$ for $i \in\{1, \ldots, h-1\}$. Therefore we have $i_{0}\left(\phi, \alpha_{n_{h}}\right)=\overline{b_{h}}=i_{0}\left(\phi, x^{l_{0}} \phi_{0}^{l_{1}} \cdots \phi_{h-2}^{l_{h-1}}\right)$. Let $c \in \mathbf{K}$ be a constant such that $i_{0}\left(\phi, \alpha_{n_{h}}-c x^{l_{0}} \phi_{0}^{l_{1}} \cdots \phi_{h-2}^{l_{h-1}}\right)>i_{0}\left(\phi, \alpha_{n_{h}}\right)=\overline{b_{h}}$. Put $\tilde{f}=$ $\phi^{n_{h}}+c x^{l_{0}} \phi_{0}^{l_{1}} \cdots \phi_{h-2}^{l_{h-1}}$. Then by Theorem $9.1 \tilde{f} \in \mathbf{K}[[x]][y]$ is an irreducible distinguished polynomial of degree $n, \overline{\operatorname{char}_{x}} \tilde{f}=\left(\overline{b_{0}}, \ldots, \overline{b_{h}}\right)$ and $\phi$ is a key polynomial of degree $\frac{n}{e_{h-1}}$ of $\tilde{f}$.

We have $i_{0}(f, x)=i_{0}(\tilde{f}, x)=n$. Let $\tilde{\alpha}_{n_{h}}=\alpha_{n_{h}}-c x^{l_{0}} \phi_{0}^{l_{1}} \cdots \phi_{h-2}^{l_{h-1}}$ and consider

$$
\begin{aligned}
i_{0}(\tilde{f}, f) & =i_{0}\left(\phi^{n_{h}}+c x^{l_{0}} \phi_{0}^{l_{1}} \cdots \phi_{h-2}^{l_{h-1}}, \phi^{n_{h}}+\alpha_{1} \phi^{n_{h}-1}+\cdots+\alpha_{n_{h}}\right) \\
& =i_{0}\left(\tilde{f}, \alpha_{1} \phi^{n_{h}-1}+\cdots+\alpha_{n_{h-1}} \phi+\tilde{\alpha}_{n_{h}}\right) \\
& \geq \inf \left\{i_{0}\left(\tilde{f}, \alpha_{1} \phi^{n_{h}-1}\right), \ldots, i_{0}\left(\tilde{f}, \alpha_{n_{h}-1} \phi\right), i_{0}\left(\tilde{f}, \tilde{\alpha}_{n_{h}}\right)\right\}
\end{aligned}
$$

since $\tilde{f}$ is irreducible. Fix $i \in\left\{1, \ldots, n_{h}-1\right\}$. Then

$$
i_{0}\left(\tilde{f}, \alpha_{i} \phi^{n_{h}-i}\right)=i_{0}\left(\tilde{f}, \alpha_{i}\right)+\left(n_{h}-i\right) i_{0}(\tilde{f}, \phi)=e_{h-1} i_{0}\left(\phi, \alpha_{i}\right)+\left(n_{h}-i\right) \overline{b_{h}}
$$


since $\phi$ is a $(h-1)$-th key polynomial of $\tilde{f}$ and $i_{0}\left(\tilde{f}, \alpha_{i}\right)=e_{h-1} i_{0}\left(\phi, \alpha_{i}\right)$ by Lemma 10.2. Using (2) and (3) we get

$$
i_{0}\left(\tilde{f}, \alpha_{i} \phi^{n_{h}-i}\right)>e_{h-1} i \frac{\overline{b_{h}}}{e_{h-1}}+\left(n_{h}-i\right) \overline{b_{h}}=n_{h} \overline{b_{h}}
$$

for $0<i<n_{h}$. Moreover, again by Lemma 10.2

$$
\text { (6) } i_{0}\left(\tilde{f}, \tilde{\alpha}_{n_{h}}\right)=e_{h-1} i_{0}\left(\phi, \tilde{\alpha}_{n_{h}}\right)>e_{h-1} i_{0}\left(\phi, \alpha_{n_{h}}\right)=e_{h-1} \overline{b_{h}} \text {. }
$$

Using (3), (5) and (6) we obtain $i_{0}(\tilde{f}, f)>e_{h-1} \overline{b_{h}}$ and the theorem follows from the Abhyankar-Moh irreducibility criterion (Corollary 8.3).

Using Proposition 10.4 and Theorem 10.5 we get a recurrent description of the class of branches with given semigroup.

Theorem 10.6 Let $\left(\overline{b_{0}}, \ldots, \overline{b_{h}}\right)$ be an $n$-characteristic sequence $(n>1)$ and let $f \in \mathbf{K}[[x]][y]$ be a distinguished polynomial of degree $n$. Then the following two conditions are equivalent

1. $f$ is irreducible and $\overline{\operatorname{char}_{x}} f=\left(\overline{b_{0}}, \ldots, \overline{b_{h}}\right)$,

2. there exists a distinguished irreducible polynomial $\phi \in \mathbf{K}[[x]][y]$ such that

$$
\begin{aligned}
& \text { (a) } \overline{\operatorname{char}_{x}} \phi=\left(\frac{\overline{b_{0}}}{e_{h-1}}, \ldots, \frac{\overline{b_{h-1}}}{e_{h-1}}\right), \\
& \text { (b) } \Delta_{x, \phi}(f)=\left\{\frac{\overline{b_{h}}}{\overline{e_{h-1}}}\right\} .
\end{aligned}
$$

To illustrate the above result let us write down

Corollary 10.7 Let $f \in \mathbf{K}[[x]][y]$ be a distinguished polynomial of degree $n>1$ and let $m>0$ be an integer such that $\operatorname{gcd}(n, m)=1$. Then $f$ is irreducible with $\overline{\operatorname{char}_{x}} f=(n, m)$ if and only if there exists a power series $\psi(x) \in \mathbf{K}[[x]], \psi(0)=0$ such that

$$
f=(y+\psi(x))^{n}+\alpha_{1}(x)(y+\psi(x))^{n-1}+\cdots+\alpha_{n}(x),
$$

where ord $\alpha_{i}>i \frac{m}{n}$ for $0<i<n$ and ord $\alpha_{n}=m$.

Example 10.8 (see [Te2], Example 4.23) Let $p=\operatorname{char} \mathbf{K} \neq 0$. Let $f=$ $y^{p}-x^{p-1}(1+y)=y^{p}-x^{p-1} y-x^{p-1}$. Using Corollary 10.7 with $\psi(x) \equiv 0$ we check that $f$ is irreducible and $\overline{\operatorname{char}_{x}} f=(p, p-1)$. 
Theorem 10.9 (Abhyankar's irreducibility criterion) Let $f \in \mathbf{K}[[x]][y]$ be a distinguished polynomial of degree $n>1$. Assume that $n \neq \equiv 0$ (mod char $\mathbf{K})$. Then $f$ is irreducible if and only if there exists an $n$-characteristic sequence $\overline{b_{0}}, \ldots, \overline{b_{h}}$ such that

1. $i_{0}\left(f, \sqrt[e_{k}-1]{f}\right)=\overline{b_{k}}$ and

2. $\Delta_{x, \sqrt{e_{k-1}}}\left(\sqrt[e_{k}]{f}\right)=\left\{\frac{\overline{b_{k}} / e_{k}}{\overline{e_{k-1} / e_{k}}}\right\}$ for $k \in\{1, \ldots, h\}$.

Proof. The conditions are necessary: if $f$ is irreducible and $\overline{\operatorname{char}_{x}} f=$ $\left(\overline{b_{0}}, \ldots, \overline{b_{h}}\right)$ then both statements hold by Theorem 6.2 and Proposition 10.3 .

The conditions are sufficient: this assertion follows from Theorem 10.5 by induction on the length $h$ of the $n$-characteristic sequence.

To check the first condition we determine the sequences $\overline{b_{0}}, \ldots, \overline{b_{h}}$ and $e_{0}, \ldots, e_{h}$ such that

- $\overline{b_{0}}=b_{0}=n$,

- $\overline{b_{k}}=i_{0}\left(f, \sqrt[e_{k-1}]{f}\right), e_{k}=\operatorname{gcd}\left(e_{k-1}, \overline{b_{k}}\right)$ for $k \in\{1, \ldots, h\}$,

- $e_{0}>\cdots>e_{h}=\operatorname{gcd}\left(e_{h}, i_{0}\left(f, \sqrt[e_{h}]{f}\right)\right)$.

The first condition holds if and only if $e_{h}=1$ and $n_{k-1} \overline{b_{k-1}}<\overline{b_{k}}$ for $k>1$.

Example 10.10 (see $[\underline{[P o}], p$. 301) Let $f=\left(y^{2}-x^{3}\right)^{2}-4 x^{5} y-x^{7}$. Assume that char $\mathbf{K} \neq 2$. Let $\overline{b_{0}}=e_{0}=4, \overline{b_{1}}=i_{0}(f, \sqrt[1]{f})=i_{0}(f, y)=6, e_{1}=$ $\operatorname{gcd}(4,6)=2, \overline{b_{1}}=i_{0}(f, \sqrt[2]{f})=i_{0}\left(f, y^{2}-x^{3}\right)=13, e_{2}=\operatorname{gcd}(2,13)=1$. The sequence $\left(\overline{b_{0}}, \overline{b_{1}}, \overline{b_{2}}\right)=(4,6,13)$ is a 4 -characteristic since $n_{1} \overline{b_{1}}=12$ is strictly less than $\overline{b_{2}}=13$. Thus condition (1) in Abhyankar's irreducibility criterion holds. To check (2) we compute

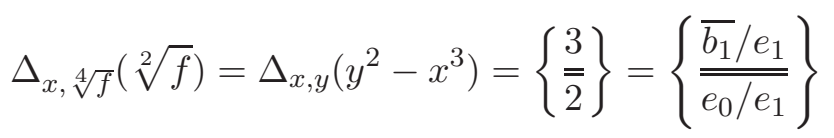

and

$$
\Delta_{x, \sqrt[2]{f}}(\sqrt[1]{f})=\Delta_{x, \sqrt[2]{f}}\left((\sqrt[2]{f})^{2}-4 x^{5} y-x^{7}\right)=\left\{\frac{13}{\overline{2}}\right\}=\left\{\frac{\overline{b_{2}} / e_{2}}{\overline{e_{1} / e_{2}}}\right\} .
$$

Therefore condition (2) holds and by Theorem $10.9 f$ is irreducible provided that char $\mathbf{K} \neq 2$. If char $\mathbf{K}=2$ then $f=y^{4}+x^{6}-x^{7}=\left(y^{2}+i x^{6}+\cdots\right)\left(y^{2}-\right.$ $\left.i x^{6}+\cdots\right)$, where $i^{2}=-1$ in $\mathbf{K}$ and $f$ is not irreducible. 


\section{Notes}

The first description of the class of branches with given semigroup is due to Teissier [Te1] (see also [CN] and [Ja]). Our approach is inspired by the papers by Abhyankar [A2 and Kuo[ $\mathrm{Ku}$ ] (see also [McCl and [As-Ba]). The generalization of the Newton polygon introduced by Kuo in $[\mathrm{Ku}$ is useful in Valuation Theory Va], Section 5. Our presentation of Abhyankar's irreducibility criterion differs from the original one. Another version of Abhyankar's criterion is due to Cossart and Moreno-Socías Co-MS1] and Co-MS2] . A criterion of irreducibility based on different ideas was given recently by [GB-G]. The $g$-adic expansions of polynomials and Newton polygons were applied to generalize the classical Shönemann-Eisenstein irreducibility criterion in the early twentieth century (see $\mathrm{O}]$ ).

\section{Merle-Granja's Factorization Theorem}

Let $\{f=0\}$ be a branch different from $\{x=0\}$. Let $\overline{\operatorname{char}_{x}} f=\left(\overline{b_{0}}, \ldots, \overline{b_{h}}\right)$, $\overline{b_{0}}=n>1$. In this section we prove the following result on factorization of power series (see [Gran] and [Mer]).

Theorem 11.1 (Merle-Granja's Factorization Theorem) Fix $k, 1 \leq$ $k \leq h$. Let $g=g(x, y) \in \mathbf{K}[[x, y]]$ be a power series such that

1. $i_{0}(g, x)=\frac{n}{e_{k}}-1$,

2. $i_{0}(f, g)=\sum_{i=1}^{k}\left(n_{i}-1\right) \overline{b_{i}}$.

Then there is a factorization $g=g_{1} \cdots g_{k} \in \mathbf{K}[[x, y]]$ such that

1. $i_{0}\left(g_{i}, x\right)=\frac{n}{e_{i}}-\frac{n}{e_{i-1}}$ for $i \in\{1, \ldots, k\}$,

2. if $\phi \in \mathbf{K}[[x, y]]$ is an irreducible factor of $g_{i}, i \in\{1, \ldots, k\}$ then

(a) $\frac{i_{0}(f, \phi)}{i_{0}(\phi, x)}=\frac{e_{i-1} \overline{b_{i}}}{n}$

(b) $i_{0}(\phi, x) \equiv 0\left(\bmod \frac{n}{e_{i-1}}\right)$.

This statement is very close to Granja's theorem (see Gran]) where the Apéry sequences are used) and is a generalization of Merle's result on polar curves. 
Remark 11.2 Let $f_{0}, \ldots, f_{h}$ be a sequence of key polynomials of $f$. Fix $k \in\{1, \ldots, h\}$. Take $g=f_{0}^{n_{1}-1} \cdots f_{k-1}^{n_{k}-1}$. Then $g$ satisfies conditions (1) and (2) of Theorem 11.1. Here $g_{i}=f_{i-1}^{n_{i}-1}$ for $i \in\{1, \ldots, k\}$.

If $\phi$ is an irreducible factor of $g_{i}$ then $\phi=f_{i-1} \cdot$ unit. Clearly $\frac{i_{0}(f, \phi)}{i_{0}(\phi, x)}=\frac{e_{i-1} \overline{b_{i}}}{n}$ and $i_{0}(\phi, x)=\frac{n}{e_{i-1}}$.

To prove Theorem 11.1 we need a few lemmas.

Lemma 11.3 Let $k$ be an integer such that $1 \leq k \leq h$. Then

1. $\left(n_{1}-1\right) \overline{b_{1}}+\cdots+\left(n_{k}-1\right) \overline{b_{k}} \not \equiv 0\left(\bmod e_{k-1}\right)$,

2. $\left(n_{1}-1\right) \overline{b_{1}}+\cdots+\left(n_{k}-1\right) \overline{b_{k}}<\overline{b_{k+1}}$,

3. if $\left(n_{1}-1\right) \overline{b_{1}}+\cdots+\left(n_{k}-1\right) \overline{b_{k}}=a_{0} \overline{b_{0}}+a_{1} \overline{b_{1}}+\cdots+a_{k} \overline{b_{k}}$ with integers $a_{0}, \ldots, a_{k}$ such that $a_{0}, a_{k} \geq 0$ and $0 \leq a_{i}<n_{i}$ for $i \in\{1, \ldots, k-1\}$ then $a_{0}=0$ and $a_{i}=n_{i}-1$ for $i \in\{1, \ldots, k\}$.

Proof. Suppose that $\left(n_{1}-1\right) \overline{b_{1}}+\cdots+\left(n_{k}-1\right) \overline{b_{k}} \equiv 0\left(\bmod e_{k-1}\right)$. Then $\left(n_{k}-\right.$ $1) \overline{b_{k}} \equiv 0\left(\bmod e_{k-1}\right)$ and $\left(n_{k}-1\right) \frac{\overline{b_{k}}}{e_{k}} \equiv 0\left(\bmod n_{k}\right)$. We get a contradiction because $\operatorname{gcd}\left(\frac{\overline{b_{k}}}{e_{k}}, n_{k}\right)=1$.

Now we will prove the second statement: if $k=h$ then $\overline{b_{k+1}}=\overline{b_{h+1}}=+\infty$ and the inequality is obvious. Let $k<h$. Then $\left(n_{1}-1\right) \overline{b_{1}}+\cdots+\left(n_{k}-1\right) \overline{b_{k}}=$ $\left(n_{1} \overline{b_{1}}-\overline{b_{1}}\right)+\cdots+\left(n_{k} \overline{b_{k}}-\overline{b_{k}}\right)<\overline{b_{k+1}}-\overline{b_{1}}<\overline{b_{k+1}}$.

To finish the proof, let $a_{k}=q n_{k}+a_{k}^{\prime}$ with $0 \leq a_{k}^{\prime}<n_{k}$. By assumption we get $q n_{k} \overline{b_{k}}=\left(-a_{0}\right) \overline{b_{0}}+\left(n_{1}-1-a_{1}\right) \overline{b_{1}}+\cdots+\left(n_{k}-1-a_{k}^{\prime}\right) \overline{b_{k}}$. The identity above is a Bézout's relation. Since $q n_{k} \overline{b_{k}} \equiv 0\left(\bmod e_{k-1}\right)$ we get by the unicity of Bézout's relation $-a_{0} \geq 0$ and $n_{k}-1-a_{k}^{\prime}=0$ that is $a_{0}=0$ and $a_{k}^{\prime}=n_{k}-1$. Therefore we get $q n_{k} \overline{b_{k}}=\left(n_{1}-1-a_{1}\right) \overline{b_{1}}+\cdots+\left(n_{k-1}-1\right) \overline{b_{k-1}} \leq$ $\left(n_{1}-1\right) \overline{b_{1}}+\cdots+\left(n_{k-1}-1\right) \overline{b_{k-1}}<\overline{b_{k}}$ by the second statement. Thus $q=0$ and again by the unicity of Bézout's relation we get the last statement.

Lemma 11.4 Let $\phi \in \mathbf{K}[[x, y]]$ be an irreducible power series such that $\frac{i_{0}(f, \phi)}{i_{0}(\phi, x)}<\frac{e_{k-1} \overline{b_{k}}}{n}$ for $a k>0$. Then $i_{0}(f, \phi) \in \overline{b_{0}} \mathbf{N}+\cdots+\overline{b_{k-1}} \mathbf{N}$.

Proof. Let $f_{k-1}$ be a $(k-1)$-th key polynomial of $f$. Thus $i_{0}\left(f_{k-1}, x\right)=$ $\frac{n}{e_{k-1}}, i_{0}\left(f, f_{k-1}\right)=\overline{b_{k}}$ and $\overline{\operatorname{char}_{x}} f_{k-1}=\left(\frac{\overline{b_{0}}}{e_{k-1}}, \ldots, \frac{\overline{b_{k-1}}}{e_{k-1}}\right)$. Since $\frac{e_{k-1} \overline{b_{k}}}{n}=$ $\frac{i_{0}\left(f, f_{k-1}\right)}{i_{0}\left(f_{k-1}, x\right)}$ we get by assumption the inequality $\frac{i_{0}(f, \phi)}{i_{0}(\phi, x)}<\frac{i_{0}\left(f, f_{k-1}\right)}{i_{0}\left(f_{k-1}, x\right)}$ that is 
$d_{x}(f, \phi)<d_{x}\left(f, f_{k-1}\right)$. Using the STI to the power series $\phi, f_{k-1}$ and $f$ we get $d_{x}(f, \phi)=d_{x}\left(\phi, f_{k-1}\right)$, which implies $i_{0}(f, \phi)=\frac{i_{0}(f, x)}{i_{0}\left(f_{k-1}, x\right)} i_{0}\left(f_{k-1}, \phi\right)=$ $e_{k-1} i_{0}\left(f_{k-1}, \phi\right) \in \overline{b_{0}} \mathbf{N}+\cdots+\overline{b_{k-1}} \mathbf{N}$.

Lemma 11.5 Let $\phi$ be an irreducible power series such that $\frac{i_{0}(f, \phi)}{i_{0}(\phi, x)}=\frac{e_{k-1} \overline{b_{k}}}{n}$ for $a k>0$. Then $i_{0}(\phi, x) \equiv 0\left(\bmod \frac{n}{e_{k-1}}\right)$ and $i_{0}(f, \phi) \equiv 0\left(\bmod \overline{b_{k}}\right)$.

Proof. Since $i_{0}(f, \phi)=\frac{e_{k-1} \overline{b_{k}}}{n} i_{0}(\phi, x)$ it suffices to check that $i_{0}(\phi, x) \equiv 0$ $\left(\bmod \frac{n}{e_{k-1}}\right)$. If $k=1$ it is clear, so assume $k>1$. We have $\frac{i_{0}(f, \phi)}{i_{0}(\phi, x)}=$ $\frac{e_{k-1} \overline{b_{k}}}{n}>\frac{e_{k-2} \overline{b_{k-1}}}{n}$ hence $i_{0}(\phi, x) \equiv 0\left(\bmod \frac{n}{e_{k}}\right)$ by Lemma 8.1,

Now we can prove the Factorization Theorem.

Proof of Theorem 11.1Let us fix a $k \in\{1, \ldots, h\}$ and let $g \in \mathbf{K}[[x, y]]$ be such that the conditions (1) and (2) hold. Let $g=\phi_{1} \cdots \phi_{s}$ with irreducible $\phi_{j} \in \mathbf{K}[[x, y]]$ for $j \in\{1, \ldots, s\}$. Firstly we check

$(*)$ if $\phi$ is an irreducible factor of $g$ then $\frac{i_{0}(f, \phi)}{i_{0}(\phi, x)} \leq \frac{e_{k-1} \overline{b_{k}}}{n}$.

Indeed, in the contrary case there would exist an irreducible factor $\phi$ of $g$ such that $\frac{i_{0}(f, \phi)}{i_{0}(\phi, x)}>\frac{e_{k-1} \overline{b_{k}}}{n}$ and we would get $i_{0}(\phi, x) \equiv 0\left(\bmod \frac{n}{e_{k}}\right)$ by Lemma 8.1. which is a contradiction since $i_{0}(\phi, x) \leq i_{0}(g, x) \leq \frac{n}{e_{k}}-1<\frac{n}{e_{k}}$.

$(* *)$ There exists (at least one) irreducible factor $\phi$ of $g$ such that $\frac{i_{0}(f, \phi)}{i_{0}(\phi, x)}=$ $\frac{e_{k-1} \overline{b_{k}}}{n}$.

If $\frac{i_{0}(f, \phi)}{i_{0}(\phi, x)} \neq \frac{e_{k-1} \overline{b_{k}}}{n}$ for all irreducible factors of $g$ then we would get by $(*)$ $\frac{i_{0}\left(f, \phi_{j}\right)}{i_{0}\left(\phi_{j}, x\right)}<\frac{e_{k-1} \overline{b_{k}}}{n}$ for all $j \in\{1, \ldots, s\}$.

By Lemma 11.4 we would have $i_{0}\left(f, \phi_{j}\right) \in \overline{b_{0}} \mathbf{N}+\cdots+\overline{b_{k-1}} \mathbf{N}$ for $j \in\{1, \ldots, s\}$ and consequently $i_{0}(f, g)=\sum_{j=1}^{s} i_{0}\left(f, \phi_{j}\right) \in \overline{b_{0}} \mathbf{N}+\cdots+\overline{b_{k-1}} \mathbf{N}$. This is impossible because $i_{0}(f, g)=\sum_{i=1}^{n}\left(n_{i}-1\right) \overline{b_{i}} \not \equiv 0\left(\bmod e_{k-1}\right)$ by the first statement of Lemma 11.3 .

Now, let us put $g_{k}$ the product of all factors $\phi_{j}$ of $g$ such that $\frac{i_{0}\left(f, \phi_{j}\right)}{i_{0}\left(\phi_{j}, x\right)}=$ $\frac{e_{k-1} \overline{b_{k}}}{n}$. Therefore we get $g=\tilde{g} g_{k}$ in $\mathbf{K}[[x, y]]$. Using Lemma 11.4 we check that $i_{0}(f, \tilde{g}) \in \overline{b_{0}} \mathbf{N}+\cdots+\overline{b_{k-1}} \mathbf{N}$ and by Lemma 11.5 we get $i_{0}\left(f, g_{k}\right) \equiv 0$ $\left(\bmod \overline{b_{k}}\right)$. 
Let us write $i_{0}(f, \tilde{g})=a_{0} \overline{b_{0}}+a_{1} \overline{b_{1}}+\cdots+a_{k-1} \overline{b_{k-1}}$ with $a_{0} \geq 0$ and $0 \leq$ $a_{i} \leq n_{i}-1$ and $i_{0}\left(f, g_{k}\right)=a_{k} \overline{b_{k}}, a_{k} \geq 0$. Therefore we get $\sum_{i=1}^{k}\left(n_{i}-1\right) \overline{b_{i}}=$ $i_{0}(f, g)=i_{0}(f, \tilde{g})+i_{0}\left(f, g_{k}\right)=a_{0} \overline{b_{0}}+a_{1} \overline{b_{1}}+\cdots+a_{k-1} \overline{b_{k-1}}+a_{k} \overline{b_{k}}$.

By the third statement of Lemma 11.3 we have $a_{0}=0$ and $a_{k}=n_{k}-1$. Thus $i_{0}\left(f, g_{k}\right)=\left(n_{k}-1\right) \overline{b_{k}}$ and $i_{0}\left(g_{k}, x\right)=\frac{n}{e_{k}}-\frac{n}{e_{k-1}}$ since $\frac{i_{0}\left(f, g_{k}\right)}{i_{0}\left(g_{k}, x\right)}=\frac{e_{k-1} \overline{b_{k}}}{n}$. If $k=1$ we are done (if $k=1$ then $i_{0}(f, \tilde{g})=0$, that is $\tilde{g}$ is a unit and we put $\tilde{g} g_{1}$ instead of $g_{1}$ ). If $k>1$ then $\tilde{g}=\frac{g}{g_{k}}$ satisfies the assumptions of Theorem 11.1 with $k-1$. We use induction on $k$.

Remark 11.6 In the proof of Merle-Granja's factorization theorem we used the inequality $i_{0}(g, x) \leq \frac{n}{e_{k}}-1$ instead of the equality $i_{0}(g, x)=\frac{n}{e_{k}}-1$. Therefore this inequality and condition (2) imply condition (1) of Theorem 11.1 .

Theorem 11.7 (Merle's factorization theorem) Suppose that $\overline{\operatorname{char}_{x}} f=$ $\left(\overline{b_{0}}, \ldots, \overline{b_{h}}\right), \overline{b_{0}}=n>1$ and $n \not \equiv 0(\bmod \operatorname{char} \mathbf{K})$. Then $\frac{\partial f}{\partial y}=g_{1} \cdots g_{h}$ in $\mathbf{K}[[x, y]]$, where

1. $i_{0}\left(g_{i}, x\right)=\frac{n}{e_{i}}-\frac{n}{e_{i-1}}$ for $i \in\{1, \ldots, k\}$.

2. If $\phi \in \mathbf{K}[[x, y]]$ is an irreducible factor of $g_{i}, i \in\{1, \ldots, h\}$, then $\frac{i_{0}(f, \phi)}{i_{0}(\phi, x)}=\frac{e_{i-1} \overline{b_{i}}}{n}$ and $i_{0}(\phi, x) \equiv 0\left(\bmod \frac{n}{e_{i-1}}\right)$.

Proof. Since $n \not \equiv 0(\bmod$ char $\mathbf{K})$ we have $i_{0}\left(\frac{\partial f}{\partial y}, x\right)=n-1$. By the Dedekind formula and the Conductor formula we have $i_{0}\left(f, \frac{\partial f}{\partial y}\right)=c(f)+$ $n-1=\sum_{k=1}^{h}\left(n_{k}-1\right) \overline{b_{k}}$. We apply Theorem 11.1 to the series $g=\frac{\partial f}{\partial y}$.

\section{Notes}

The first result on factorization of the derivative was proved by Henry J.S. Smith in $\mathrm{Sm}$ but his work fell into oblivion for a long time. Merle proved the factorization theorem in the generic case, the observation that the theorem is true in any coordinates is due to Ephraim [E]. Granja's theorem is formulated in terms of Apéry sequences and proved using the HamburgerNoether expansions (see Gran]).

Acknowledgements: The authors are very grateful to Bernard Teissier for reading the manuscript and making valuable suggestions. 


\section{References}

[A1] Abhyankar, S.S. Local analytic geometry. Pure and Applied Mathematics, XIV Academic Press, New York 1964 xv+484 pp.

[A2] Abhyankar, S.S. Irreducibility criterion for germs of analytic functions of two complex variables. Adv. Math. 74 (1989), no. 2, 190-257.

[A3] Abhyankar, S.S. Expansion techniques in Algebraic Geometry. Tata Institute of Fundamental Research Lectures on Mathematics and Physics, 57. Tata Institute of Fundamental Research, Bombay, 1977. iv+168 pp.

[A-M1] Abhyankar, S.S.; Moh, T.T. Newton-Puiseux expansion and generalized Tschirnhausen transformation. I, II. J. reine angew. Math. 260 (1973), 47-83; ibid. 261 (1973), 29-54.

[A-M2] Abhyankar, S.S.; Moh, T.T. Embeddings of the line in the plane. J. reine angew. Math. 276 (1975), 148-166.

[Anc] Ancochea Quevedo, G. Curvas algebraicas sobre cuerpos cerrados de característica cualquiera. Memorias de la Real Academia de Ciencias Exactas, Físicas y Naturales de Madrid. Serie de Ciencias exactas. Tomo IV. Memoria n. 1.

[Ang] Angermüller, G. Die Wertehalbgruppe einer ebener irreduziblen algebroiden Kurve. Math. Z. 153 (1977), no. 3, 267-282.

[As-Ba] Assi, A; Barile, M. Effective construction of irreducible curve singularities. Int. J. Math. Comp. Sci. 1 (2006), no. 1, 125-149.

[Az] Azevedo, A. The jacobian ideal of a plane algebroid curve. Thesis. Purdue University, Indiana. 1967.

[Bre] Bresinsky, H. Semigroups corresponding to algebroid branches in the plane. Proc. Amer. Math. Soc. 32 (1972), no. 2, 381-384.

[Cam1] Campillo, A. Algebroid curves in positive characteristic. Lecture Notes in Mathematics, 813. Springer Verlag, Berlin, 1980. v+168 pp.

[Cam2] Campillo, A. Hamburger-Noether expansions over rings. Trans. Amer. Math. Soc. 279 (1983), no. 1, 377-388. 
[CN] Cassou-Noguès, P. Courbes de semi-groupe donné. Rev. Mat. Univ. Complut. Madrid 4 (1991), no. 1, 13-44.

[Chad-Pł] Chądzyński, J.; Płoski, A. An inequality for the intersection multiplicity of analytic curves. Bull. Polish Acad. Sci. Math. 36 (1988), no. 3-4, 113-117.

[Chan-W] Chang, H. C.; Wang, L. C. An intersection-theoretical proof of the embedding line theorem. J. Algebra 161 (1993), no. 2, 467479 .

[Chan] Chang, H. C. On equisingularity, analytical irreducibility and embedding line theorem. Chinese J. Math. 19 (1991), no. 4, 379389 .

[Co-MS1] Cossart, V.; Moreno-Socías, G. Irreducibility Criterion: A Geometric Point of View. Valuation theory and its applications, Vol. II (Saskatoon, SK, 1999), 27-42, Fields Inst. Commun., 33, Amer. Math. Soc., Providence, RI, 2003.

[Co-MS2] Cossart, V.; Moreno-Socías, G. Racines approchées, suites génératrices, suffisance des jets. Ann. Fac. Sci. Toulouse Math. (6) 14 (2005), no. 3, 353-394.

[Del] Delgado de la Mata, F. A factorization theorem for the polar of a curve with two branches. Compositio Math. 92 (1994), no. 3, $327-375$.

[E] Ephraim, R. Special polars and curves with one place at infinity. Singularities, Part 1 (Arcata, Calif., 1981), 353-359, Proc. Sympos. Pure Math., 40, Amer. Math. Soc., Providence, RI, 1983.

[GB] García Barroso, E. Courbes polaires et courbure des fibres de Milnor des courbes planes. PhD thesis. Université Paris 7 Denis Diderot (2000).

[GB-G] García Barroso, E.; Gwoździewicz, J. Characterization of jacobian Newton polygons of plane branches and new criteria of irreducibility. Ann. Inst. Fourier (Grenoble) 60 (2010), no. 2, 683709.

[Gar-St] García, A.; Stöhr, K.O. On semigroups of irreducible algebroid plane curves. Comm. Algebra 15 (1987), no. 10, 2185-2192. 
[Gran] Granja, A. Apéry basis and polar invariants of plane curve singularities. Pacific J. Math. 140 (1989), no. 1, 85-96.

[Gw-Pł] Gwoździewicz, J.; Płoski,A. On the approximate roots of polynomials. Ann. Polon. Math. 60 (1995), no. 3, 199-210.

[Ja] Jaworski, P. Normal forms and bases of local rings of irreducible germs of functions of two variables. Trudy Sem. Petrovsk. no. 13 (1988), 19-35, 256; translation in J. Soviet Math. 50 (1990), no. $1,1350-1364$.

[He] Hefez, A. Irreducible plane curve singularities. Real and complex singularities, 1-120, Lecture Notes in Pure and Appl. Math., 232, Dekker, New York, 2003.

[J] Jung, H. W. E. Über ganze birationale Transformationen der Ebene. J. reine angew. Math. 184 (1942). 161-174.

[Ku] Kuo, T.C. Generalized Newton-Puiseux theory and Hensel's lemma in C[[x,y]]. Canad. J. Math. 41 (1989), no. 6, 1101-1116

[LJ] Lejeune-Jalabert, M. Sur l'équivalence des courbes algébroïdes planes. Coefficients de Newton. Contribution à l'etude des singularités du poit du vue du polygone de Newton, Paris VII, Janvier 1973, Thèse d'Etat.

See also in Travaux en Cours, 36 (edit. Lê Dũng Trãng) Introduction à la théorie des singularités I, 49-124, 1988.

[Mac] MacLane, S. A construction for absolute values in polynomials rings. Trans. Amer. Math. Soc. 40 (1936), no. 3, 363-395.

[McC] McCallum, S. On testing a bivariate polynomial for analytic reducibility. J. Symbolic Comput. 24 (1997), no. 5, 509-535.

[Mer] Merle, M. Invariants polaires des courbes planes. Invent. Math. 41 (1977), no. 2, 103-111.

[Moh1] Moh, T.T. On characteristic pairs of algebroid plane curves for characteristic p. Bull. Inst. Math. Acad. Sinica 1 (1973), no. 1, 75-91.

[Moh2] Moh, T.T. On analytic irreducibility at $\infty$ of a pencil of curves. Proc. Amer. Math. Soc. 44 (1974), 22-24. 
[N] Nagata, M. A theorem of of Gutwirth. J. Math. Kyoto Univ. 11 (1971), 149-154.

[O] Ore, O. Zur Theorie der Irreduzibilitätskriterien. Math. Zeit. 18(1923), 278-288.

[Pi] Pinkham, H. Courbes planes ayant une seule place a l'infini, Séminaire sur les Singularités des surfaces, Centre de Mathématiques de l'École Polytechnique, Année 1977-1978.

[Pł] Płoski, A. Remarque sur la multiplicité d'intersection des branches planes. Bull. Polish Acad. Sci. Math. 33 (1985), no. 11-12, 601-605.

[Po] Popescu-Pampu, P. Approximate roots. Valuation theory and its applications, Vol. II (Saskatoon, SK, 1999), 285-321, Fields Inst. Commun., 33, Amer. Math. Soc., Providence, RI, 2003.

[Re] Reguera López, A. Semigroups and clusters at infinitiy. Algebraic geometry and singularities (La Rábida, 1991), 339-374, Progr. Math., 134, Birkhäuser, Basel, 1996.

[Ru] Russell, P. Hamburger-Noether expansions and approximate roots of polynomials. Manuscripta Math. 31 (1980), no. 1-3, 2595.

[Sa-St] Sathaye, A.; Stenerson, J. Plane polynomial curves. Algebraic geometry and its applications (West Lafayette, IN, 1990), 121142, Springer, New York, 1994.

[Sei1] Seidenberg, A. Valuation ideals in polynomial rings. Trans. Amer. Math. Soc. 57, (1945). 387-425.

[Sei2] Seidenberg, A. Elements of the theory of algebraic curves. Addison-Wesley Publishing Co., Reading, Mass.- London-Don Mills, Ont. 1968 viii+216 pp.

[Sm] Smith, H.J.S. On the higher singularities of plane curves. Proc. London Math. Soc. s1-6(1) (1875), 153-182.

[Sp] Spivakovsky, M. Valuations in function fields of surfaces. Amer. J. Math. 112 (1990), no. 1, 107-156.

[vdK] van der Kulk, W. On polynomial rings in two variables. Nieuw Arch. Wiskunde (3) 1, (1953) 33-41. 
[Va] Vaquié, M. Valuations. Resolution of singularities (Obergurgl, 1997), 539-590, Progress in Math., 181, Birkhäuser, Basel, 2000.

[Te1] Teissier, B. Appendix in [Za3].

[Te2] Teissier, B. Valuations, deformations, and toric geometry. Valuation theory and its applications, Vol. II (Saskatoon, SK, 1999), 361-459, Fields Inst. Commun., 33, Amer. Math. Soc., Providence, RI, 2003.

[Te3] Teissier, B. Complex curve singularities: a biased introduction. Singularities in geometry and topology, 825-887, World Sci. Publ., Hackersanck, NJ, 2007.

[Za1] Zariski, O. Studies in equisingularity. I. Equivalent singularities of plane algebroid curves. Amer. J. Math. 87 (1965), 507-536.

[Za2] Zariski, O. General theory of saturation and of saturated local rings. II. Saturated local rings of dimension. Amer. J. Math. 93 (1971), 872-964.

[Za3] Zariski, O. Le problème des modules pour les branches planes. Centre de Mathématiques de l'École Polytechnique, Paris, 1973. With an appendix by Bernard Teissier. Second edition. Hermann, Paris, 1986. x+212 pp.

Evelia Rosa García Barroso

Departamento de Matemática Fundamental

Facultad de Matemáticas, Universidad de La Laguna

38271 La Laguna, Tenerife, España

e-mail: ergarcia@ull.es

Arkadiusz Płoski

Department of Mathematics

Technical University

Al. 1000 L PP7

25-314 Kielce, Poland

e-mail: matap@tu.kielce.pl 